\title{
Atmospheric chemosynthesis is phylogenetically and geographically widespread and contributes significantly to carbon fixation throughout cold deserts
}

\author{
Angelique Ray \\ University of New South Wales \\ Julian Zaugg
}

Australian Centre for Ecogenomics, School of Chemistry and Molecular Biosciences, The University of Queensland, St Lucia, Queensland, Australia

Nicole Benaud

University of New South Wales

Devan Chelliah

University of New South Wales

\section{Sean Bay}

Monash University https://orcid.org/0000-0003-4833-3106

Hon Lun Wong

UNSW Sydney https://orcid.org/0000-0002-3801-0410

\section{Pok Man Leung}

Monash University https://orcid.org/0000-0002-5382-827X

\section{Mukan Ji}

Chinese Academy of Sciences

\section{Aleks Terauds}

Australian Antarctic Division

\section{Kate Montgomery}

University of New South Wales

Chris Greening

Monash University https://orcid.org/0000-0001-7616-0594

\section{Don Cowan}

University of Pretoria

\section{Weidong Kong}

Institute of Tibetan Plateau Research https://orcid.org/0000-0001-9682-1484

\section{Timothy Williams}

University of New South Wales

Philip Hugenholtz 
The University of Queensland https://orcid.org/0000-0001-5386-7925

Belinda Ferrari ( $\square$ b.ferrari@unsw.edu.au )

UNSW Sydney https://orcid.org/0000-0001-5043-3726

\section{Article}

Keywords: Atmospheric chemosynthesis, carbon fixation, photosynthesis, trace gases, RuBisCO, cold desert, soil, microbiomes

Posted Date: October 13th, 2021

DOl: https://doi.org/10.21203/rs.3.rs-948000/v1

License: (9) This work is licensed under a Creative Commons Attribution 4.0 International License. Read Full License 


\section{Abstract}

Cold desert soil microbiomes thrive despite severe moisture and nutrient limitations. In Eastern Antarctic soils, hydrogen oxidising bacteria support primary production through a novel carbon fixation process reliant on the chemoautotrophy-associated RuBisCO form IE. Here, biochemical assays show that atmospheric chemosynthesis occurs globally for primary production, contributing significantly to autotrophic carbon fixation throughout arid to hyperarid deserts in Antarctica, the high Arctic, and the Tibetan Plateau. Taxonomic and functional analyses were performed on 230 dereplicated medium-tohigh quality metagenome-assembled genomes (MAGs) derived from 18 cold desert metagenomes and an additional 24,080 publicly available genomes. We infer that atmospheric chemosynthetic bacteria are widespread across environmental and clinical samples, increasing our knowledge of the bacterial phyla genetically capable of atmospheric chemosynthesis to seven, with key enzymes co-occurring within MAGs from four previously unidentified phyla; Chloroflexota, Firmicutes, Deinococcota and Verrucomicrobiota. We informatically identify an additional group of high-affinity hydrogenases, group $1 \mathrm{~m}$ [NiFe]-hydrogenase using phylogenetics, gene structure analysis and homology modelling and reveal substantial new genetic diversity within RuBisCO form IE ( $r b c L 1 E)$, and high-affinity groups $1 \mathrm{~h}$ and $1 \mathrm{l}$ [NiFe]-hydrogenases. Finally, we conclude that atmospheric chemosynthesis is a global phenomenon, extending throughout and beyond cold deserts, with significant implications for the global carbon cycle and bacterial survival within environmental and clinical reservoirs.

\section{Main Text}

Microbial communities inhabiting cold desert soils thrive ${ }^{1-5}$ despite limited exposure to liquid water, scarce organic and inorganic edaphic resources, frequent freeze-thaw cycles and, particularly in the polar latitudes, extreme and variable exposure to sunlight and UV radiation ${ }^{6-9}$. Polar desert soils are usually oligotrophic and often contain limited photosynthetic taxa, resulting in a need to supplement energy and organic carbon inputs through alternative autotrophic processes beyond photosynthesis and 'traditional' chemoautotrophy 2,10-12. The microbial oxidation of atmospheric molecular hydrogen $\left(\mathrm{H}_{2}\right)$ and carbon monoxide (CO) gases are well known processes that provide soil bacteria with a reliable and ubiquitous source of energy to persist ${ }^{13-22}$. However, the oxidation of these trace gases has also been linked to an under-investigated chemoautotrophic primary production process known as atmospheric chemosynthesis, which supports microbial carbon inputs in extreme terrestrial environments where photosynthetic input is low ${ }^{11,14,23-25}$. During atmospheric chemosynthesis, trace levels of $\mathrm{CO}$ and $\mathrm{H}_{2}$ are oxidised using type 1 [MoCu]-CO dehydrogenases (CODH) and high-affinity group $1 \mathrm{~h}$-[NiFe]-hydrogenases ( $h h y L$ ), respectively. Additional high-affinity assimilatory hydrogenases, specifically group $1 \mathrm{~L}$ [NiFe]hydrogenase $(h y / L){ }^{14,23}$ and group 2a [NiFe]-hydrogenase $(h u c L){ }^{20}$, have recently been discovered and may also contribute to this process. Electrons yielded from atmospheric $\mathrm{H}_{2}$ and $\mathrm{CO}$ oxidation act in conjunction with ribulose-1,5-biphosphate carboxylase/oxygenase (RuBisCO) form IE (rbcL 1E), supporting carbon fixation through the Calvin-Benson-Bassham (CBB) cycle ${ }^{11,14}$. The genes encoding RuBisCO form IE and high-affinity hydrogenase are ubiquitous and in high abundance across oligotrophic 
deserts spanning the Antarctic, Arctic and Tibetan Plateau ${ }^{12}$, although activity studies confirming expression of the atmospheric chemosynthetic pathway are limited. While nineteen bacterial and six archaeal phyla contain trace gas oxidisers that use the energy derived from aerobic respiration to support persistence ${ }^{14,26}$, thus far only three phyla have been proposed to be capable of atmospheric chemosynthesis; Actinobacteriota, Candidatus Dormibacterota and Ca. Eremiobacterota ${ }^{11}$.

Here, we use metagenomics, phylogenetics and biochemical approaches to quantify the contribution of atmospheric chemosynthesis to primary production in soils from cold deserts that span the globe, including New Harbour, Taylor Valley, Mitchell Peninsula, Windmill Islands and The Ridge, Vestfold Hills, in Antarctica; the Ngari Prefecture in the Qinghai-Tibet Plateau in China; and Spitsbergen, Svalbard and Alexandra Fjord Highlands in the high Arctic. Genome-resolved metagenomics was used to determine autotrophic capacities within our 18 soil metagenomes and 230 dereplicated MAGs, as well as 24,080 reference genomes from the Genome Taxonomy Database (GTDB) originating from a broad range of environments, including the human body. We substantially expand upon the known diversity of the RuBisCO form IE gene, identifying representatives in eight bacterial phyla, which co-occur with trace gas oxidation genes in not only environmental but also clinical samples.

\section{Trace gas chemotrophic phyla dominate cold desert soil microbiomes}

Shotgun sequencing produced approximately 8-10 Gb metagenomes from each of the 18 Antarctic, Arctic, and Tibetan Plateau soil samples (Supplementary 1). Analysis of prokaryotic marker genes in these metagenomes indicated that the bacterial and archaeal communities comprised 48 phyla and 127 classes (Supplementary 3). Consistent with previous studies, polar soils were dominated by Actinobacteriota, Proteobacteria, Chloroflexota, and Acidobacteriota, with Gemmatimonadota, Bacteroidota and Verrucomicrobiota also prevalent in the High Arctic and some Antarctic sites (Figure 1, Supplementary 3 and 4) 2,23,27,28. Phyla associated with atmospheric chemosynthetic potential, including Actinobacteriota dominated the Antarctic and Tibetan Plateau soil samples, accounting for up to $83 \%$ of the microbial community in The Ridge samples (Supplementary 3), while Ca. Dormibacterota (av 12.3\%) and Ca. Eremiobacterota (av 5.6\%) were abundant in Mitchell Peninsula samples (Figure 1, Supplementary 3,4$)$. By contrast, the photosynthetic Cyanobacteria were rare, accounting for an average relative abundance of $<0.57 \%$ across Antarctic and Tibetan Plateau soils. Comparatively, Actinobacteriota were less abundant in high Arctic soils (18.1-29.6\%) (Supplementary 3), which exhibited a higher abundance of Cyanobacteria compared with the Antarctic sites (Spitsbergen Svalbard S 0.1 $0.8 \%$ and Alexandra Fjord Highlands 1.3-2.3\%) (Figure 1, Supplementary 3). Eukaryotic taxa were limited within all soil metagenomes (average $<0.012 \%$ ) and were dominated by Ascomycota and Basidiomycota (Supplementary 5). Photosynthetic eukaryotic phyla, specifically Chlorophyta, Cryptophyta, Ochrophyta and Rhodophyta, occurred at very low relative abundances $(<0.0013 \%)$ (Supplementary 5$)$.

Assembly and binning of all 18 metagenomes yielded 230 high or medium-quality ( $50 \%$ completeness and $10 \%$ contamination) MAGs after dereplication at the species level ( $\leq 95 \% \mathrm{ANI})$ ). Of these, 76 were estimated to be more than $90 \%$ complete and $<5 \%$ contaminated (Supplementary 2 ). The MAGs 
encompassed 16 bacterial and 1 archaeal phyla, with the obtained taxa reflecting metagenomic profiles with Actinobacteriota $(n=86)$, Chloroflexota $(n=23)$, Proteobacteria $(n=21)$, Acidobacteriota $(n=20)$, Bacteroidota $(n=18)$, Verrucomicrobiota $(n=11)$ and Gemmatimonadota $(n=10)$ being the most dominant (Supplementary 2). Archaeal MAGs were solely obtained from the phylum Thermoproteota $(n=7)$ (Supplementary 2). Several recently proposed bacterial candidate phyla were also represented by MAGs including Ca. Eremiobacterota ${ }^{29}, \mathrm{Ca}$. Dormibacterota ${ }^{30}, \mathrm{Ca}$. Sumerlaeota ${ }^{31}$ and $\mathrm{Ca}$. Patescibacteria ${ }^{32,33}$ as well as two MAGs from the metabolically flexible predatory phylum Bdellovibrionota (Supplementary 2) ${ }^{34,35}$. MAGs spanned known autotrophic phyla including those capable of atmospheric chemosynthesis (Actinobacteriota, $\mathrm{Ca}$. Eremiobacterota and $\mathrm{Ca}$. Dormibacterota) $(n=96),{ }^{11}$, oxygenic photosynthesis (Cyanobacteria) $(n=2)$ and anoxygenic photosynthesis (Proteobacteria; orders Rhizobiales, Rhodobacterales and Burkholderiales) $(n=6)^{36}$.

Photoautotrophic green sulfur bacteria belonging to the class Chlorobi were notably undetected in both the MAGs and metagenomes, as were photoheterotrophic taxa including the genera Candidatus Chloracidobacterium, Heliobacterium, and Gemmatimonas ${ }^{36-41}$. Chloroflexia, the sole photoautotrophic class within Chloroflexota ${ }^{42}$, was undetected. The only Chloroflexota MAGs represented belonged to the chemoautotrophic order Thermomicrobiales $(n=7)$, which is associated with $\mathrm{CO}$ oxidation ${ }^{18,43}$, and the poorly characterised candidate order 54-19 $(n=2)$.

\section{Photoautotrophic and geochemical-driven chemoautotrophic capacities are limited in arid and hyperarid polar soil microbiomes}

To understand the autotrophic strategies sustaining life in cold desert ecosystems, we explored the potential for carbon and nitrogen cycling within soil metagenomes and MAGs. Genetic markers of aerobic and anaerobic respiration were ubiquitous in samples from all six deserts and in the recovered MAGs (Figures 2 and 3, Supplementary 7). Genes required for lithoautotrophic processes driven by the metabolisation of edaphic materials were detected in only a limited number of MAGs (Supplementary 79), These genes included those associated with the oxidation of reduced inorganic sulfur compounds through the thiosulfate oxidation pathways ( $S O x$ ) and the reverse dissimilatory sulfite reductase pathway (dsrC, dsrEFH) (Supplementary 8$)^{44,45}$. Together, this suggests a low capacity for the oxidation of geochemical substrates to support microbial carbon fixation throughout these ecosystems.

Genes associated with ammonia oxidation ( $a m o A$, hao) were uncommon and genes associated with nitrite-oxidation (nxrAB) were not detected in any MAGs (Supplementary 9). There was some evidence for biological nitrogen fixation across all six deserts. Consistent with the low Cyanobacteria abundances and low nitrogen-fixation potential, nitrogenase gene ( $(\mathrm{nifH})$ abundances were also low in the metagenomes (0.49-3.7\%) and were undetected within the recovered MAGs (Supplementary 9 and 10). Ammonia monooxygenase $(a m o A)$, associated with nitrification, was uncommon in all metagenomes $(<1.3 \%)$ especially in the Antarctic (average $0.13 \%$ ) (Supplementary 10) and were almost exclusively detected in the ammonia oxidising archaea (Thermoproteota) MAGs (Supplementary 9), a common feature in 
environmentally constrained Antarctic soils ${ }^{28,46-49}$. Denitrification capacities were widespread, with copper-containing nitrite reductase (nirK) generally more prevalent than nirS in both the MAGs (Figure 4, Supplementary 9) and the metagenomes (Supplementary 10). Most MAGs encode a wide range of carbohydrate-active enzymes (CAZys) that are predicted to hydrolyse hydrocarbons, starch, hemicellulose, chitin and oligosaccharides (Supplementary 11 and 16). This suggests a capacity for organotrophy and the use of sugar-containing biopolymers as carbon and energy sources. Notably, such organic substrates are limited in oligotrophic desert soils, especially in Antarctic regions where plant matter is generally limited to moss and lichens ${ }^{50,51}$, consistent with the very low eukaryotic signal in all metagenomes studied (relative average abundance < $0.012 \%$ ) (Supplementary 5). It is possible that these hydrolytic genes and pathways are largely inactive; however, they indicate the prevalence of taxa capable or both heterotrophy and autotrophy, consistent with previous studies of terrestrial desert microbiomes ${ }^{11,14,52,53}$.

Genes associated with photosystems I ( $p s a A-F, p s a l-M, p s a X)$ and II ( $p s b A-F, p s b H-M, p s b O P, p s b T-Z$, $p s b 27, p s b 28, p s b 28-2)$ were detected solely within the two Cyanobacteria MAGs found exclusively within Alexandra Fjord Highland samples, whilst photosynthetic reaction centre genes commonly associated with Chlorobi ( $p s c A-D)$ were not detected (Supplementary 2 and 7). In comparison, anoxygenic photosynthesis genes (pufBA-LMC) were detected in 17 MAGs spanning the Acidobacteriota, Bacteroidota, Myxococcota and Proteobacteria (Supplementary 2 and 7). In addition to these reaction centre genes, we investigated genes encoding light-harvesting complexes and antenna proteins, which play an important role in the absorption of light for photosynthesis. Phycobilisome genes were detected within a single Chloroflexota MAG present in Mitchell Peninsula soil (average 4.13\%) (Supplementary 2 and 7), and in both Cyanobacteria MAGs present in Alexandra Fjord Highlands at relative abundances < 0.14\% (Supplementary 2 and 7). Light-harvesting complex I (LHCA1-5) and II (LHCB1-7) and chlorophyll $\mathrm{a} / \mathrm{b}$ binding light-harvesting proteins $(p c b A-H)$ were not detected in any of the MAGs or metagenomes (Supplementary 7). Genes for the protochlorophyllide complex subunits, $b c h N$ and $b c h B$, were found in MAGs of the Rubrobacteraceae family of Actinobacteriota; but in members of Rubrobacteraceae these genes do not appear to be connected to bacteriochlorophyll-based phototrophy ${ }^{54,55}$. MAGs assigned to the genus Amaricoccus (Rhodobacteraceae, Alphaproteobacteria) contained pufL, pufM, and $b c h B$ genes although RuBisCO genes were undetected (Supplementary 2 and 7). Indicating the potential for photoheterotrophy, proteorhodopsin genes with DTE motifs were identified in our Bacteroidota MAGs, whilst the Deinococcota MAGs harbored a higher variety of rhodopsins, including halorhodopsins with TSV and TSD motifs, xenorhodopsin with DTA motif and an outward $\mathrm{H}+$ pumping rhodopsin with a DTK motif (Supplementary 17A). All rhodopsins except xenorhodopsin harbours DxxxK motif on the 7th transmembrane helix (TM7), indicating retinal binding properties (Supplementary 17B). Xenorhodopsin in Deinococcota (NH3_7) has a NxxxK motif on TM7, which has not been previously reported. Together, these results indicate a limited, but potentially significant capacity for microbial phototrophy, particularly within the Alexandra Fjord Highlands and Mitchell Peninsula soil microbiomes. 


\section{Trace gas oxidation supports maintenance and productivity in arid and hyperarid ecosystems across the poles}

Our analysis of gene diversity suggest that phototrophy and the oxidation of geochemical compounds have a limited capacity to support the electron transport chain and drive carbon fixation within the polar desert microbiomes studied here. Conversely, there was an extensive genetic capacity for trace gas oxidation, with high-affinity [NiFe]-hydrogenases from group $1 \mathrm{~h}, 1 \mathrm{l}$ and 2 a distributed across all 18 soil metagenomes (Figure 2, Supplementary 10), with either groups $1 \mathrm{~h}$ or $1 \mathrm{l}$ detected in a third of MAGs ( $50 \%$ completeness, $10 \%$ contamination) (Figure 3, Supplementary 6). To our knowledge, this is the first identification of a group 11 [NiFe]-hydrogenase in the phyla Deinococcota (order Deinococcales) (Bin\#: $160,161)$ and further supports the presence of this hydrogenase in the phyla Actinobacteriota, Bacteroidota, Chloroflexota, and Proteobacteria ${ }^{23}$ (Supplementary 6). MAGs containing high affinity group $1 \mathrm{~h}$ [NiFe]-hydrogenase genes were found in seven previously established trace gas oxidising phyla: Acidobacteriota, Actinobacteriota, Chloroflexota, Ca. Dormibacterota, Ca. Eremiobacterota, Proteobacteria, and Verrucomicrobiota 11,14,56,57 (Figure 3 and 4, Supplementary 6). Phylogenetic analysis revealed a novel clade of [NiFe]-hydrogenases (Supplementary 13), hereby referred to as group $1 \mathrm{~m}$. Like groups $1 \mathrm{~h}$ and $1 \mathrm{l}$ [NiFe]-hydrogenase, group $1 \mathrm{~m}$ was detected in all metagenomes and a further 13 of the obtained MAGs, all of which are members of Actinobacteriota.

Genetic analysis showed structural similarities between $1 \mathrm{~m}$ [NiFe]-hydrogenase and the wellcharacterised high-affinity $1 \mathrm{~h}$ [NiFe]-hydrogenase, as well as the newly discovered high-affinity $1 \mathrm{ll}$ [NiFe]hydrogenase, implying that $1 \mathrm{~m}$ [NiFe]-hydrogenases is also a high-affinity enzyme. Simultaneously, key differences that justify classification of these sequences into a novel grouping were consistently observed. For example, like the $1 \mathrm{~h}$ [NiFe]-hydrogenase gene cluster, the small and large subunits of $1 \mathrm{~m}$ [NiFe]-hydrogenase were encoded by adjacent genes. This contrasts with the form 1 l [NiFe]-hydrogenase gene cluster which, consistent with a previous study ${ }^{23}$, has five short predicted transmembrane proteins (HylTM1-5) interposing the large and small subunits. The group $1 \mathrm{~m}$ [NiFe]-hydrogenase gene cluster typically also contained the following genes, most of which are unique to this novel group: HybD peptidase involved in processing of the hydrogenase large subunit ${ }^{58}$; an FeS cluster assembly protein; a $\mathrm{Ni}$ insertion ATPase/GTPase (CooC-type); tetratricopeptide repeat (TPR) protein (in general involved in assembly of multiprotein complexes); a bifunctional ligase/repressor (BirA) homolog; a DUF1059 domain (of unknown function); and two small proteins (82-92 amino acids) with no identifiable domains that each contain a single transmembrane helix (Supplementary 19). The group $1 \mathrm{~h}$ [NiFe]-hydrogenase from C. nectator H16 (PDB ID = 5AA5) ${ }^{59}$ was consistently identified as the best model for the $1 \mathrm{~m}$ [NiFe]hydrogenase amino acid sequences extracted from our MAGs (Phyre2 ${ }^{53}$; hhyL 87-89\% residues modelled at $>90 \%$ confidence, $h$ hmS $68-79 \%$ residues modelled at $>90 \%$ confidence; SWISS-MODEL ${ }^{60}$ Global Model Quality Estimate $=0.67-0.72$ for the whole tetramers). From this informatic analysis, we conclude that $1 \mathrm{~m}$ [NiFe]-hydrogenase is an evolutionarily and structurally distinct group of high-affinity enzymes. 
In terms of $\mathrm{CO}$ oxidation, proteins annotated as the aerobic carbon monoxide dehydrogenase large subunit (coxL) were highly prevalent across the 230 MAGs. However, as most MAGs lacked the catalytic cluster of $\mathrm{CODH}^{61}$, we infer that only 18 of the 230 MAGs contained genes for the complete CODH protein. These included members of the Actinobacteriota, Chloroflexota, and Ca. Dormibacterota (Supplementary 6).

RuBisCO form IE accounted for $68 \%$ of all RuBisCO genes identified in the metagenomes, dominating in samples from the Antarctic sites; Mitchell Peninsula, The Ridge and New Harbour (average relative abundances of $18 \%, 17 \%$ and $8.1 \%$, respectively), and occurring in lower abundances in Tibetan Plateau, Spitsbergen Svalbard and Alexandra Fjord Highland samples (3.1\%, 1.9\% and 1.4\%, respectively; Figure 2, Supplementary 10). Although widely distributed, the photosynthetic RuBisCO forms IA and 1B were comparatively present only in low abundance in all metagenomes (average $1.1 \%$ and $0.2 \%$, respectively).

RuBisCO form IE was encoded in 38 of the obtained MAGs, while RuBisCO forms II, III, IA, IB, IC and ID were collectively limited to only 5 MAGs (Figure 3, Supplementary 6). Overall, 25 MAGs contained both a detectable RuBisCO form IE and high-affinity hydrogenase genes (Figure 4, Supplementary 6). Of these, 18 belonged to the phylum Actinobacteriota, three to the phylum Chloroflexota (family Ktedonobacteraceae including novel genera UBA11361 and CF-113) and two each to the established trace gas chemosynthetic phyla $\mathrm{Ca}$. Dormibacterota and Ca. Eremiobacterota ${ }^{62,63}$. Of these 25 MAGs, eight also encoded CODH with an active-site loop ${ }^{61}$, The eight MAGs containing all three genes ( $r b c L 1 E$, hhyL/hylL/ hhmL and CODH) belong to the Actinobacteriota (order Mycobacteriales including novel genus QHCD01, and order Solirubrobacterales including novel genus Palsa-465), Chloroflexota (two novel genera in the family Ktedonobacteraceae, CF-113 and UBA11361), and Ca. Dormibacterota (genus Candidatus Dormibacter) (Figure 4, Supplementary 2, 6 and 7). Therefore, these taxa potentially possess high metabolic flexibility, with the potential to use $\mathrm{H}_{2}, \mathrm{CO}$ and $\mathrm{CO}_{2}$ for both hydrogenotrophic and carboxydotrophic growth (Figure 4, Supplementary 6 and 7).

Interestingly, only a single MAG (classified as Actinobacteriota, family Solirubrobacteraceae) encoded both CODH and RuBisCO form IE but no detectable high-affinity hydrogenase gene. This suggests that in cold desert microorganisms, $\mathrm{CO}$ oxidation is rarely the sole driver of atmospheric chemosynthesis, occurring most frequently in conjunction with $\mathrm{H}_{2}$ oxidation.

RuBisCO form IE was also detected in 12 MAGs where neither CODH or a high-affinity hydrogenase were detected, including those belonging to Actinobacteriota (including novel families SIRW01, AC-14 and HRBIN12 and novel genus GCA-003244245) and Chloroflexota (including novel order 54-19 and novel family UBA6265) (Supplementary 2, 6 and 7). Whilst this might be a result of incomplete MAG assembly or annotation, it is also possible that RuBisCO form IE activity can be driven by alternative energy sources besides atmospheric $\mathrm{H}_{2}$ or $\mathrm{CO}$ oxidation. Further biochemical studies of these taxa in isolation would likely clarify the intriguing role of alternative energy sources in this process. 
Atmospheric chemosynthesis and photosynthesis co-occur in microbial communities to support primary production

Gas chromatography was used to confirm scavenging and oxidation of atmospheric $\mathrm{H}_{2}$ and $\mathrm{CO}$ in soil microcosms of all six global desert sites. Headspace $\mathrm{H}_{2}$ concentrations rapidly dropped to subatmospheric levels (Figure 5A, Supplementary 14), with average atmospheric hydrogen oxidation rates ranging from $9.4 \mathrm{nmol} / \mathrm{mol} / \mathrm{h} / \mathrm{g}$ at The Ridge through to $421.4 \mathrm{nmol} / \mathrm{mol} / \mathrm{h} / \mathrm{g}$ at Mitchell Peninsula. Antarctic and Tibetan Plateau soil microcosms demonstrated the highest $\mathrm{H}_{2}$ oxidation rates, consistent with the higher abundances of Actinobacteriota within these samples (Figure 1, Supplementary 3, 4 and 13). Mitchell Peninsula microcosms demonstrated extremely rapid $\mathrm{H}_{2}$ oxidation, likely reflecting the high abundances of the phyla $\mathrm{Ca}$. Eremiobacterota and $\mathrm{Ca}$. Dormibacterota (average 7.8\% and 3.6\%, respectively) that are capable of atmospheric chemosynthesis (Figure 1, Supplementary 3, 4 and 13). The rapid $\mathrm{H}_{2}$ uptake rates reported here far exceed those previously calculated to be required to sustain the energy needs of similarly structured polar and temperate terrestrial microbiomes $11,14,33,64-67$, such as those from Robinson Ridge $(3.49 \mathrm{nmol} / \mathrm{mol} / \mathrm{h} / \mathrm{g})$ and Adams Flat $(5.54 / \mathrm{nmol} / \mathrm{mol} / \mathrm{h} / \mathrm{g})$ soils in Eastern Antarctica ${ }^{11}$, as well as cultured bacterial isolates ${ }^{68}$.

$\mathrm{CO}$ oxidation was also observed in soil microcosms from all desert sites except The Ridge (Figure 5B). However, the rates observed were much slower than for $\mathrm{H}_{2}$ oxidation in the same microcosms, and high levels of variation in $\mathrm{CO}$ oxidation rates were observed between soils from the same site (Supplementary 14). These results, combined with the greater abundance of putative $\mathrm{H}_{2}$ oxidising bacteria compared to putative $\mathrm{CO}$ oxidisers in the soil samples, suggests that atmospheric $\mathrm{H}_{2}$ oxidation is more important and widespread energy acquisition process (c.f., $\mathrm{CO}$ oxidation) in these polar soil microbiomes.

We also demonstrated that atmospheric chemosynthesis contributes to primary production in globallydistributed cold desert soils (Figure 6). The assimilation of ${ }^{14} \mathrm{CO}_{2}$ was consistently higher in New Harbour $(p=0.013)$, Alexandra Fjord Highlands $(p=0.031)$ and Spitsbergen Svalbard $(p=0.033)$ soil microcosms with $\mathrm{H}_{2}$ supplementation compared to those without $\mathrm{H}_{2}$ addition. In the high Arctic desert soils, light exposure also led to a significant increase ( $p=0.015$ and $p=0.022$, respectively) in carbon fixation, suggesting the co-occurrence of atmospheric chemosynthesis and photosynthesis. Genes encoding elements of the photosynthetic apparatus were also routinely detected in the metagenomes (Figure 3 , Supplementary 7). Higher rates of photosynthesis within the high Arctic sites are likely attributed to a higher abundance of photosynthetic taxa, particularly Cyanobacteria in the Alexandra Fjord Highlands (Figure 1, Supplementary 3). Consistent with previous studies ${ }^{69-71}$, greater edaphic Cyanobacteria abundances are likely a reflection of greater moisture availability at these sites $(0.077 \%$ Alexandra Fjord Highlands, $0.243 \%$ SS) ${ }^{12}$. Furthermore, this may in turn account for a lower relative abundance of chemoautotrophic and desiccation-tolerant taxa, particularly Actinobacteria, within the Arctic microbiomes ${ }^{71,72}$. Conversely, light exposure did not stimulate significant ${ }^{14} \mathrm{CO}_{2}$ fixation rates in Mitchell Peninsula soils, although both variabilities in ${ }^{14} \mathrm{CO}_{2}$ assimilation rates and bacterial community structure 
were observed for the three Mitchell Peninsula soils examined. For example, MP3 was comprised of a higher abundance of photosynthetic Cyanobacteria (1.1\% relative abundance), with higher levels of ${ }^{14} \mathrm{CO}_{2}$ fixation in the presence of light observed in contrast to MP1 and MP2 soils $(0.2 \%$ and $0.4 \%$ relative Cyanobacteria abundance, respectively) (Figure 1, Supplementary 3). It should be noted that in MP3 higher Cyanobacteria abundances co-occurred with higher relative abundances of atmospheric chemosynthetic phyla Ca. Eremiobacterota (5.6\%) and Ca. Dormibacterota (12.3\%). In MP2, low photosynthetic potential combined with a high relative abundance of $\mathrm{Ca}$. Eremiobacterota (5.1\%) and Ca. Dormibacterota (10.9\%) was found, whilst photosynthetic and atmospheric chemosynthetic potential were both low in MP1 (0.2\% Ca. Eremiobacterota) and ( $0.3 \% \mathrm{Ca}$. Dormibacterota). With such great taxonomic variation between biological replicates, it is unsurprising that inconsistent biochemical activity was observed.

\section{RuBisCO form 1E phylogeny spans eight bacterial phyla inhabiting environmental and clinical reservoirs}

To support the analysis of our MAGs, we extracted a further putative 4,507 RuBisCOs, 1,073 high-affinity hydrogenases and 1,289 aerobic carbon monoxide dehydrogenases based on sequence identity ( $>30 \%$ to representative sequences, $70 \%$ alignment) from 24,080 bacterial and archaeal representative genomes from release R04-R89 of the GTDB. Phylogenetic analyses confirmed the identity of the extracted RuBisCO sequences (190 form IE, 291 form ID, 251 form IC, 194 form IB, 423 form IA, 348 form II, 275 form III, and 1188 form IV) (Supplementary 15) and high-affinity hydrogenase groups (722 form 1h, 6 form 1m, 19 form 1I) (Supplementary 13). Consistent with analysis of our own MAGs, RuBisCO form IE was widely distributed, found within Actinobacteriota, Chloroflexota, $\mathrm{Ca}$. Dormibacterota and $\mathrm{Ca}$. Eremiobacterota (Figure 4, Supplementary 6) and, in accordance with previous studies ${ }^{11,73}$, Firmicutes and Verrucomicrobiota (Figure 4).

High-affinity group 1h-[NiFe]-hydrogenases co-occurred with RuBisCO form IE within MAGs from each of the six trace gas assimilating phyla detected, whilst CODH with an active-site loop co-occurred with RuBisCO form IE in all phyla except Verrucomicrobiota (Figure 4, Supplementary 2, 6 and 7). Analysis of the GTDB genomes revealed the first examples of RuBisCO form IE within Acidobacteriota $(n=5)$ and Deinococcota $(n=14)$ MAGs, although group 1 [NiFe]-hydrogenase was not detected within either (Figure 4). Of these MAGs, four Deinococcota harboured a $\operatorname{cox} \mathrm{L}$ gene that coded for a protein with a CODH active-site loop (Figure 4), suggesting a capacity for atmospheric chemosynthesis using carboxydotrophy rather than hydrogenotrophy. Therefore, in addition to Actinobacteriota, $\mathrm{Ca}$. Dormibacterota and $\mathrm{Ca}$. Eremiobacterota, the bacterial phyla Chloroflexota, Deinococcota, Firmicutes and Verrucomicrobiota are now implicated as being capable of atmospheric chemosynthesis through $\mathrm{H}_{2}$ and/or $\mathrm{CO}$ oxidation.

Notably, 80 of the GTDB reference genomes that contained both RuBisCO form IE and group $1 \mathrm{~h}$ [NiFe]hydrogenase genes were obtained from the analysis of pure cultured microorganisms, rather than MAGs. The Actinobacteriota accounted for most of these isolates $(n=76)$ (orders Mycobacteriales, Streptomycetales, Streptosporangiales and Solirubrobacterales), with Firmicutes (Sulfobacillus thermosulfidooxidans), Chloroflexota (Nitrolancea hollandica) and Verrucomicobiota (Methylacidiphilum kamchatkense) also represented. Both Firmicutes and 35 of the 76 Actinobacteriota genomes also 
contained a catalytic CODH unit with an active-site loop. In addition to the 80 isolates described, a further 12 reference genomes obtained from microbial isolates contained RuBisCO form IE and a catalytic CODH unit but did not have a detectable group 1 [NiFe]-hydrogenase gene, suggesting a propensity for atmospheric chemosynthesis through carboxydotrophic rather than hydrogenotrophic growth. These isolates also spanned Actinobacteriota and Firmicutes and included a third phylum; Deinococcota (Meiothermus cerbereus). The discovery of atmospheric chemosynthetic genes within already isolated microorganisms opens the possibility for more extensive culture-dependent research that could conclusively characterise the activation conditions and mechanisms of the underlying atmospheric chemosynthetic pathways.

Overall, 92 GTDB reference genomes obtained from bacterial isolates are inferred to have the capacity for atmospheric chemosynthesis by harbouring genes for RuBisCO form IE and either aerobic CODH or group $1 \mathrm{~h}$ [NiFe]-hydrogenase or both. These bacteria have been previously isolated from a broad range of temperate and desert environments, the majority from soil. Soil environments included agricultural fields ${ }^{74,75}$, karst caves ${ }^{76,77}$, plant matter ${ }^{78,79}$ and mining and ore deposits ${ }^{80}$. These putative atmospheric chemosynthetic bacteria were also isolated from aqueous environments, including ocean ${ }^{81}$, sediment ${ }^{82-84}$, lakes ${ }^{85}$, river and stream ${ }^{86,87}$, hot spring ${ }^{88,89}$ and groundwater samples ${ }^{90}$. Interestingly, aqueous environments such as these generally have a lower capacity for oxygenation compared to terrestrial systems, highlighting the need to confirm activation of the atmospheric chemosynthetic pathway in these organisms and characterise it in greater detail.

A number of Actinobacteriota that encoded RuBisCO form IE and trace gas scavenging enzymes were also isolated from human-host tissue samples, including skin lesions ${ }^{91}$, sputum ${ }^{92-95}$, lymph nodes ${ }^{96}$, the eye ${ }^{97}$, gastrointestinal tract ${ }^{98}$ and haemodialysis water ${ }^{99}$. This is perhaps not surprising given that opportunistic pathogens within the Mycobacterium and Rhodococcus genera are capable of oxidising CO and/or $\mathrm{H}_{2}$ at below atmospheric levels. This diverse habitat range supports the hypothesis that highaffinity $\mathrm{H}_{2}$ and $\mathrm{CO}$ oxidation is globally pervasive, potentially a diverse range of microorganisms in many different habitats with multiples mechanisms to acquire energy to support survival and growth.

\section{Conclusion}

Atmospheric chemosynthesis supplements photosynthetic primary production in cold desert soils across the globe, with trace gas oxidisation providing the energy and/or carbon needs to sustain terrestrial ecosystems in the high Arctic, Antarctica, and Tibetan Plateau. This observation expands the significance of soil microorganisms as key elements in the global carbon budget. We have informatically identified an additional novel high-affinity hydrogenase, termed $1 \mathrm{~m}$-[NiFe]-hydrogenase, and increased the list of bacterial phyla capable of atmospheric chemosynthesis to seven, with key enzymes co-occurring within MAGs from four previously unidentified phyla; Chloroflexota, Firmicutes and Deinococcota and Verrucomicrobiota. The discovery of a suite of putative trace gas chemotrophs from diverse habitats, including potentially anoxic sediments, highlights their proposed metabolic flexibility - being capable of 
mixotrophic growth and persistence during periods of nutrient-starvation. Finally, the isolation of presumptive trace gas chemotrophs from a range of environmental and clinical reservoirs provides new opportunities to experimentally demonstrate and confirm the underlying metabolic pathways of atmospheric chemosynthesis and to clarify the physiological importance of the energy generation and carbon fixation processes in bacterial survival.

\section{Materials And Methods}

\section{Site descriptions}

Two Eastern Antarctic sites were chosen for inclusion in this study: Mitchell Peninsula (MP) $\left(66^{\circ} 31^{\prime} \mathrm{S}\right.$, $\left.110^{\circ} 59^{\prime} \mathrm{E}\right)$ from the Windmill Islands region and The Ridge (TR) $\left(68^{\circ} 54^{\prime} \mathrm{S}, 78^{\circ} 07^{\prime} \mathrm{E}\right)$ from the Vestfold Hills

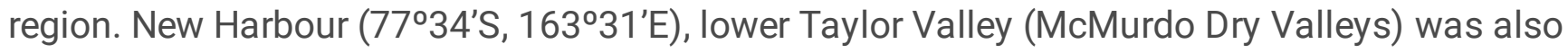
investigated, as were two high Arctic sites; Alexandra Fjord Highlands (Alexandra Fjord Highlands) $\left(78^{\circ} 51^{\prime} \mathrm{N}, 75^{\circ} 54^{\prime} \mathrm{W}\right)$ in Canada and Spitsbergen Svalbard $\left(78^{\circ} 14^{\prime} \mathrm{N}, 15^{\circ} 25^{\prime} \mathrm{W}\right)$ in Norway. Samples were also collected from the cold, high-altitude Qinghai-Tibet Plateau in Western China $\left(32^{\circ} 27^{\prime} \mathrm{N}, 80^{\circ} 4^{\prime} \mathrm{E}\right)$.

\section{Soil sampling}

Sampling of Mitchell Peninsula, The Ridge, Alexandra Fjord Highlands and Spitsbergen Svalbard was conducted under auspices of the Australian Antarctic Program (AAP). New Harbour was sampled by the Centre for Microbial Ecology and Genomics, University of Pretoria, and the Tibetan Plateau soils were obtained from the Institute of Tibetan Plateau Research, Chinese Academy of Sciences. Three surface soils were collected from each of these sites (total samples; 18). All soil samples were stored at $-80{ }^{\circ} \mathrm{C}$ until used in this study.

\section{Community DNA extraction and sequencing}

DNA was extracted in triplicate from $0.25-0.30 \mathrm{~g}$ of each soil sample using the FastDNA SPIN kit for soil (MP Biomedicals, NSW, Australia) as per the manufacturer's instructions. Metagenomic shotgun libraries were prepared from DNA extractions using the Nextera XT DNA Sample Preparation Kit (Illumina Inc.). Sequencing was performed on an Illumina NextSeq500 platform with $2 \times 150$ base pair high output run chemistry and $7 \mathrm{~Gb}$ coverage per sample.

\section{Metagenome assembly and binning}

Low quality reads were identified and removed with Trimmomatic ${ }^{100}$ using a sliding window of 4 bases with an average quality of 21 (SLIDINGWINDOW:4:21), with those reads less than $50 \mathrm{bp}$ in length discarded (MINLEN:50). Quality controlled reads were then assembled using MEGAHIT (ver. 1.2.2beta) ${ }^{101}$ with default parameters. Contig statistics including assembly size, number of contigs, contig length distribution, and N50/90 values were calculated with BBMap (ver. 38.41) ${ }^{102}$ and custom scripts. Quality controlled reads for each sample were mapped onto their respective assemblies with minimap2 
as part of CoverM 'make' (ver. 0.3.0, B. Woodcroft, unpublished, https://github.com/wwood/CoverM). Low quality mappings were removed with CoverM 'filter' (minimum identity $95 \%$ and minimum aligned length of $75 \mathrm{bp}$ ). Assemblies for each sample were binned by providing the contigs for each sample and filtered BAM files as input to UniteM (ver. 0.0.15; D. parks, unpublished, https://github.com/dparks1134/UniteM) and using a minimum contig length of 1500bp and Maxbin (ver. 2.2.4) ${ }^{103}$, MetaBAT (ver. 0.32.5) ${ }^{104}$ and MetaBAT2 (ver. 2.12.1) ${ }^{105}$ binning methods ( $\max 40, \max 107, \mathrm{mb} 2, \mathrm{mb}$ _verysensitive, mb_sensitive, mb_specific, mb_veryspecific and mb_superspecific).

Bin completeness and contamination was evaluated using CheckM (ver. 1.0.12) ${ }^{106}$ and the taxonomy assigned using the Genome Taxonomy Database Toolkit (GTDB-Tk; ver. 1.3.0; with reference to GTDB R05-RS95) ${ }^{107}$. Binning yielded 860 bins (17 archaeal and 811 bacterial), 282 of which were complete with $10 \%$ contamination. A non-redundant set of bins were obtained by dereplicating with $\underline{\mathrm{dRep}}$ (ver. 2.2.3, sa $=0.95)^{108}$. Following dereplication, 230 metagenome-assembled genome (MAG) bins (7 archaeal and 223 bacterial; $50 \%$ completeness, $10 \%$ contamination) were selected for further analysis. Of the 230 MAGs, 76 were estimated to be more than $90 \%$ complete and less than $5 \%$ contaminated (Supplementary 2).

\section{Calculation of MAG abundances}

To calculate the relative abundance of each MAG, reads from each sample were mapped to the set of MAGs using CoverM 'make'. Low quality mappings were removed with CoverM 'filter' (minimum identity $95 \%$ and minimum aligned length of $75 \mathrm{bp}$ ). The mean coverage of each MAG was calculated with CoverM, with those with a fraction of coverage less than $5 \%$ reported as having zero coverage. The relative abundance of each MAG, among those obtained, was calculated as its coverage divided by the total summed coverage of all MAGs. Abundance values were multiplied by the fraction of reads that mapped to all MAGs to produce the relative abundance of each MAG within the entire sample.

\section{Custom database generation and metabolic annotation of metagenomic short reads}

The abundance of 43 genes, representing the forms of RuBisCO (IA, IB, IC, ID, IE, II, III and IV) and highaffinity [NiFe]-hydrogenase ( $1 \mathrm{~h}, 1 \mathrm{l}, 1 \mathrm{~m}$ and $2 \mathrm{a})$, were determined for each metagenome. Custom databases for each gene were first generated by downloading representative (seed) sequences for RuBisCO_large from Pfam ${ }^{109}$ (PF00016) and group 1h-[NiFe]-hydrogenase large subunit from the HydDB ${ }^{57}$. Potential RuBisCO and hydrogenase sequences were then identified in representative genomes from the GTDB (R04-RS89) by first predicting the gene protein-coding sequences (CDS) in each genome using Prodigal (ver. 2.6.3) as part of Prokka (ver. 1.12) and then aligning the resulting protein sequences against the representative RuBisCO_large and group $1 \mathrm{~h}$-[NiFe]-hydrogenase large subunit sequences with blast+ (ver. 2.9.0; -max_hsps 1) ${ }^{110}$. BLAST results were filtered to those hits where at least $30 \%$ sequence identity and $70 \%$ alignment of the representative sequence was achieved and false-positives manually removed. Separate phylogenetic analysis was performed upon the filtered RuBisCO and hydrogenase sequences alongside representative sequences. RuBisCO subtypes were identified according to the clades 
formed and compiled into separate protein databases. Hydrogenase sequences that clustered with the group $1 \mathrm{~h}$, group $1 \mathrm{l}$, group $1 \mathrm{~m}$ and group 2a-[NiFe]-hydrogenases clade (Supplementary 13) were also compiled into separate protein databases. An additional 35 protein databases were generated using sequences retrieved from UniprotKB protein database (March 2020) ${ }^{111}$ (Supplementary 16). These protein sequences included those associated with the CBB cycle, energy metabolism, nitrogen cycling, photosynthesis, the rTCA cycle and trace gas oxidation.

Gene abundances were then calculated by aligning reads against each of the 43 reference protein databases using DIAMOND BlastX ${ }^{112}$, with a query coverage of $80 \%$ and an identity threshold of $50 \%$. To account for differences in sample sequencing depth and gene length, read counts were normalized to reads per kilobase per million (RPKM) and further normalized against the mean RPKM value estimated from 14 single-copy ribosomal marker genes derived from SingleM (ver.

0.13.0; unpublished, https://github.com/wwood/singlem) and phylosift ${ }^{113}$ (Supplementary 10) to infer the percentage of the community encoding the gene ${ }^{23}$. These results are provided in Supplementary 10 and were also visualised as a heatmap using the R package ggplot2 ${ }^{114}$ (Figure 2).

\section{Community taxonomic profiling of the metagenomes}

The taxonomic profile of each unassembled metagenome was determined through the classification of reads corresponding to the universal single-copy ribosomal marker protein L16/L10E rp/P (Lan et al., 2016) using the approach described by Ortiz, et al. ${ }^{23}$. Briefly, rp/P sequences for representative bacterial and archaeal genomes in the GTDB R05-RS95 were downloaded (https://data.ace.uq.edu.au/public/gtdb/data/releases/release95/95.0/). GraftM (ver. 0.12.2) (Boyd et al., 2018) was then used to create a gene family specific phylogenetic package, which was used to create a classification package for SingleM (ver. 0.13.2). An operational taxonomic unit (OTU) profile was then generated using SingleM 'pipe' on the paired reads for each sample. This taxonomic profile was visualised as a bar chart at the phyla-level using the R package ggplot2 ${ }^{114}$ (Figure 1). To complement the marker gene profiling, metagenomes were also taxonomically profiled by mapping paired reads against the representative GTDB genomes with minimap2 ${ }^{115}$ as part of CoverM 'make' (ver. 0.4.0) (Supplementary 4). Low quality mappings were removed with CoverM 'filter' (minimum identity $95 \%$ and minimum aligned length of $75 \%$ ). Forward reads that remained unmapped or were filtered by CoverM were profiled by Kaiju (ver. 1.7.3) using the $n r$ teuk database ${ }^{116}$. All taxonomic profiles constructed are described at a class level (Supplementary 3-5).

\section{Gene extraction and functional annotation of MAGs}

MAGs were translated and functionally annotated using a combination of Prokka (ver. 1.14), Prodigal (ver. 2.6.3), the carbohydrate-active enzymes database (CAZy) ${ }^{117}$, and EnrichM (ver. 0.4.15, J. Boyd, unpublished, https://github.com/geronimp/enrichM), the latter using annotation options --ko_hmm, -pfam, -tigrfam, -orthologs, -clusters, -cazy and -ec. In EnrichM, for a query gene to be considered for annotation, the minimum fraction aligning to a reference, and vice versa, was set to 0.5 , with a minimum 
percent identity of $30 \%$ also required. Sequences initially annotated as CODH large subunit (coxL) were manually inspected for the presence of the CODH active-site loop ${ }^{61}$ to determine if they were likely to be $\operatorname{coxL}$; using this approach, the majority of $\operatorname{cox} L$ homologs were removed from further consideration. Rhodopsin amino acid sequences were identified through annotation against the Pfam ${ }^{109}$ database using InterProScan ${ }^{118}$, and TMHMM ${ }^{119}$ used to subsequently confirm the presence of seven transmembrane helices. Rhodopsin sequences were then aligned with MAFFT v.7.407 120,121 and visualised with Geneious Prime 2021.0.1 (https://www.geneious.com). Rhodopsin motifs were identified in the 3rd transmembrane helix while the retinal binding motifs were identified in the 7th transmembrane helix.

\section{Phylogenetic Analysis of RuBisCO and hydrogenase within MAGs and GTDB Genomes}

Potential RuBisCO and high-affinity group 1 [NiFe]-hydrogenase large subunit sequences were identified in the 860 MAGs obtained in this study and 24,080 representative genomes from the GTDB R04-RS89 using blast+ (ver. 2.9.0; -max_hsps 1) ${ }^{110}$. BLAST results were filtered to those hits where at least $30 \%$ sequence identity and $70 \%$ alignment of the representative sequence was achieved and false-positives manually removed. Phylogenetic analysis was conducted to identify the subtype of each RuBisCO and hydrogenase sequence extracted (Figure 4, Supplementary 13 and 15). In total, 4622 putative RuBisCO sequences were obtained; 115 from our constructed MAGs and 4507 from representative genomes from the GTDB. In addition, 1377 putative hydrogenase sequences were obtained; 304 from our MAGs and 1073 from representative genomes. Identical sequences were removed from further analysis.

Separate phylogenetic analyses were performed upon the extracted RuBisCO and hydrogenase sequences. Multiple sequence alignment was performed using MAFFT v.7.407, employing the L-INS-i iterative refinement method ${ }^{120,121}$. The resulting alignments were then trimmed to remove poorly aligned regions using trimAl v.1.4.1, with a gap threshold of $0.5^{122}$. Sequences with more than $50 \%$ gaps after alignment were removed. Maximum likelihood phylogenetic trees were constructed using IQ-Tree v.1.6.10 ${ }^{123}$, applying 1000 ultrafast bootstrap iterations, hill-climbing nearest neighbour interchange (NNI) search, and incorporating additional SH-like approximate likelihood ratio tests (SH-alrt) ${ }^{124}$. ModelFinder was performed to determine the best phylogenetic model, which was the amino-acid exchange rate general matrix (LG) plus 'FreeRate' model heterogeneity (+R9 for the RuBisCO tree and $+\mathrm{R} 10$ for the hydrogenase tree) ${ }^{123}$. Sequences that failed the chi2 test during tree building were removed. The final consensus trees comprised 3255 RuBisCO sequences and 2103 hydrogenase sequences and were both uploaded to iTOL ${ }^{125}$ for visualisation. Branches within the hydrogenase tree were colour-coded according to the form of hydrogenase and bootstrap values 90-100 indicated by circles on the corresponding branches. Within the RuBisCO tree, sequences were colour-coded by phyla, and branches colour-coded by RuBisCO form. MAGs were colour-coded according to the cold-desert site where they were primarily detected. Representative genomes and MAGs harbouring high-affinity hydrogenase and aerobic carbon monoxide dehydrogenase sequences were marked with triangles. A complete tree depicting all 3255 RuBisCO sequences is provided (Supplementary 15), as is a pruned version focussing 
upon the entire RuBisCO form IE clade, the 115 RuBisCO sequences extracted from the cold desert MAGs and their closest corresponding references sequences within genomes from the GTDB (Figure 4). Copy numbers obtained for the MAGs were converted to presence/absence, and the proportion of MAGs from each phyla containing the genes visualised as a heatmap using the R package ggplot2 ${ }^{114}$ (Figure 3 ).

\section{Gene structural analysis and homology modelling of 1m-[NiFe]-hydrogenase}

Amino acid sequences encoding the $1 \mathrm{~m}$ [NiFe]-hydrogenase small subunit ( $h \mathrm{hm} S$ ) and large subunits ( $h h m L)$, as well as the surrounding genes were extracted from four of the MAGs assembled in this study (bin \#: 90, 63, 45, 35). All sequences were submitted to ExPASy BLAST (using the 'UniProtKB/Swiss-Prot only' option) ${ }^{126}$ and to InterProScan ${ }^{127}$ to identify functional domains, and potential subcellular location (e.g., transmembrane helices). Amino acid sequences encoding the $1 \mathrm{~m}$ [NiFe]-hydrogenase small and large subunits from all the 4 MAGs were also modelled through input into the Protein Homology/analogY Recognition Engine V 2.0 (Phyre2) using the intensive modelling mode ${ }^{53}$. The amino acid sequence of the group $1 \mathrm{~m}$ [NiFe]-hydrogenase large and small subunits ( $h h m S L$ ) from each of the four MAGs were input into SWISS-Model ${ }^{60}$, to visualise the structure of the group $1 \mathrm{~m}$ [NiFe]-hydrogenase tetramer.

\section{$\mathrm{H}_{2}$ Oxidation and ${ }^{14} \mathrm{CO}_{2}$ Fixation Assays}

Gas chromatography was used to measure the activity of high-affinity hydrogenases and carbon monoxide dehydrogenases within microbial communities from each cold desert region. To determine whether the microbial communities within each soil sample were fixing carbon through atmospheric chemosynthesis, photosynthesis, a combination of both or neither, ${ }^{14} \mathrm{CO}_{2}$ assimilation assays were conducted. Both assays and the subsequent statistical analysis of the results were conducted according to previously described methods ${ }^{11}$ (Supplementary 18 ).

\section{Data Availability Statement}

Next generation sequencing data that supports the findings of this study have been deposited in GenBank with the accession code PRJNA664610. All other data supporting the findings of this study are available in the article/supplementary information.

\section{Declarations}

\section{Author Contributions}

B.C.F. determined the research objective with input from A.E.R and P.H. A.E.R conducted the $\mathrm{H}_{2}$ and $\mathrm{CO}$ oxidation assays and ${ }^{14} \mathrm{CO}_{2}$ assimilation assays with input from S.B. and P.L. and equipment provided by C.G. A.E.R completed the data analysis and visualisation of these assays. W.K. and M.J. provided the Tibetan Plateau soil samples, A.T. provided the The Ridge soil samples, and D.A.C. provided the New Harbour soil samples. A.E.R assembled the metagenomes with guidance from H.L.W. and J.Z. J.Z. 
conducted taxonomic analysis of the metagenomes, assembled, taxonomically classified, functionally annotated, and calculated the abundance of each MAG, with guidance from P.H. A.E.R. visualised these outputs. N.B conducted phylogenetic analysis of the RuBisCO subtypes with input from A.E.R. and A.E.R. conducted phylogenetic analysis of high-affinity hydrogenases. K.M. first identified a potential for novel hydrogenases within this work and conducted preliminary analysis. T.W. searched CODH sequences for an active-site loop and confirmed the identities of RuBisCO and hydrogenase sequences whilst H.L.W. analysed the rhodopsin sequences. D.S.C. and A.E.R. constructed custom databases and D.S.C. used them for the functional annotation and analysis of the unbinned metagenomes, which was subsequently visualised by A.E.R. H.L.W. analysed the CaZy output. T.W. conducted gene structural analysis and A.E.R. conducted subsequent homology modelling. A.E.R. and B.C.F. wrote the manuscript with input from remaining authors; all authors have read and approved the manuscript for submission.

\section{Acknowledgements}

We thank the Australian Antarctic Program expedition teams between 2012 and 2019 for sampling of the Antarctic and Arctic soils used in this study, especially Dan Wilkins, Cath King and Mark Raymond. We thank J. Gao and G. Guo for assisting soil sampling of the Tibetan Plateau. This work was supported by the Australian Government Research Training Program (RTP) Scholarship (awarded to A.R. and D.S.C.), the Australian Research Council Future Fellowship (FT170100341; awarded to B.C.F.), the Australian Antarctic Program Project 5097, the Australian Antarctic Science project grant (4406; awarded to B.C.F.), an ARC DECRA Fellowship (DE170100310; awarded to C.G.), and a NHMRC New Investigator Grant (APP5191146; awarded to C.G.). This research includes computations using the computational cluster Katana supported by Research Technology Services at UNSW Sydney.

\section{References}

1 Cowan, D. A., Makhalanyane, T. P., Dennis, P. G. \& Hopkins, D. W. Microbial ecology and biogeochemistry of continental Antarctic soils. Frontiers in microbiology 5, 154, doi:10.3389/fmicb.2014.00154 (2014).

2 Leung, P. M. et al. Energetic Basis of Microbial Growth and Persistence in Desert Ecosystems. 5, e00495-00419, doi:doi:10.1128/mSystems.00495-19 (2020).

3 Kleinteich, J. et al. Pole-to-Pole Connections: Similarities between Arctic and Antarctic Microbiomes and Their Vulnerability to Environmental Change. 5, doi:10.3389/fevo.2017.00137 (2017).

4 Neufeld, J. D. \& Mohn, W. W. Unexpectedly high bacterial diversity in arctic tundra relative to boreal forest soils, revealed by serial analysis of ribosomal sequence tags. Applied and environmental microbiology 71, 5710-5718, doi:10.1128/aem.71.10.5710-5718.2005 (2005). 
5 Tindall, B. J. Prokaryotic diversity in the Antarctic: the tip of the iceberg. Microbial ecology 47, 271283, doi:10.1007/s00248-003-1050-7 (2004).

6 Lambrechts, S., Willems, A. \& Tahon, G. Uncovering the Uncultivated Majority in Antarctic Soils: Toward a Synergistic Approach. 10, doi:10.3389/fmicb.2019.00242 (2019).

$7 \quad$ Pearce, D. A. in Adaption of Microbial Life to Environmental Extremes: Novel Research Results and Application (eds Helga Stan-Lotter \& Sergiu Fendrihan) 87-118 (Springer Vienna, 2012).

8 Alsop, T. in Encyclopedia of World Climatology (ed John E. Oliver) 651-655 (Springer Netherlands, 2005).

$9 \quad$ Fahey, D. et al. (World Meteorological Organization, 2018).

10 Bay, S., Ferrari, B. \& Greening, C. Life without water: How do bacteria generate biomass in desert ecosystems? Microbiology Australia 39, 28-32, doi:10.1071/MA18008 (2018).

$11 \mathrm{Ji}, \mathrm{M}$. et al. Atmospheric trace gases support primary production in Antarctic desert surface soil. Nature 552, 400-403, doi:10.1038/nature25014 (2017).

12 Ray, A. E. et al. Soil Microbiomes With the Genetic Capacity for Atmospheric Chemosynthesis Are Widespread Across the Poles and Are Associated With Moisture, Carbon, and Nitrogen Limitation. 11, doi:10.3389/fmicb.2020.01936 (2020).

13 Lennon, J. T. \& Jones, S. E. Microbial seed banks: the ecological and evolutionary implications of dormancy. Nature Reviews Microbiology 9, 119-130, doi:10.1038/nrmicro2504 (2011).

14 Bay, S. et al. Chemosynthetic and photosynthetic bacteria contribute differentially to primary production across a steep desert aridity gradient. The ISME Journal, doi:10.1038/s41396-021-01001-0 (2021).

15 Greening, C., Berney, M., Hards, K., Cook, G. M. \& Conrad, R. A soil actinobacterium scavenges atmospheric H\&lt;sub\&gt;2\&lt;/sub\&gt; using two membrane-associated, oxygen-dependent [NiFe] hydrogenases. Proceedings of the National Academy of Sciences 111, 4257, doi:10.1073/pnas.1320586111 (2014).

16 Greening, C. et al. Persistence of the dominant soil phylum \&lt;em\&gt;Acidobacteria\&lt;/em\&gt; by trace gas scavenging. Proceedings of the National Academy of Sciences 112, 10497, doi:10.1073/pnas.1508385112 (2015).

17 Cordero, P. R. F. et al. Atmospheric carbon monoxide oxidation is a widespread mechanism supporting microbial survival. Isme J 13, 2868-2881, doi:10.1038/s41396-019-0479-8 (2019). 
18 Islam, Z. F. et al. Two Chloroflexi classes independently evolved the ability to persist on atmospheric hydrogen and carbon monoxide. The ISME Journa/ 13, 1801-1813, doi:10.1038/s41396-0190393-0 (2019).

19 Constant, P., Poissant, L. \& Villemur, R. Isolation of Streptomyces sp PCB7, the first microorganism demonstrating high-affinity uptake of tropospheric H-2. Isme J 2, 1066-1076, doi:10.1038/ismej.2008.59 (2008).

20 Islam, Z. F. et al. A widely distributed hydrogenase oxidises atmospheric $\mathrm{H} 2$ during bacterial growth. The ISME Journal, doi:10.1038/s41396-020-0713-4 (2020).

21 Constant, P. et al. Genome data mining and soil survey for the novel group 5 [NiFe]-hydrogenase to explore the diversity and ecological importance of presumptive high-affinity H2-oxidizing bacteria. $\mathbf{7 7}$, 6027-6035 (2011).

22 Constant, P., Chowdhury, S. P., Pratscher, J. \& Conrad, R. J. E. m. Streptomycetes contributing to atmospheric molecular hydrogen soil uptake are widespread and encode a putative high-affinity [NiFe]hydrogenase. 12, 821-829 (2010).

23 Ortiz, M. et al. A genome compendium reveals diverse metabolic adaptations of Antarctic soil microorganisms. (2020).

24 King Gary, M. Contributions of Atmospheric $\mathrm{CO}$ and Hydrogen Uptake to Microbial Dynamics on Recent Hawaiian Volcanic Deposits. Applied and environmental microbiology 69, 4067-4075, doi:10.1128/AEM.69.7.4067-4075.2003 (2003).

25 Lynch, R. C., Darcy, J. L., Kane, N. C., Nemergut, D. R. \& Schmidt, S. K. Metagenomic evidence for metabolism of trace atmospheric gases by high-elevation desert Actinobacteria. 5, doi:10.3389/fmicb.2014.00698 (2014).

26 Greening, C. et al. Genomic and metagenomic surveys of hydrogenase distribution indicate $\mathrm{H} 2$ is a widely utilised energy source for microbial growth and survival. The ISME journal 10, 761-777, doi:10.1038/ismej.2015.153 (2016).

27 Cary, S. C., McDonald, I. R., Barrett, J. E. \& Cowan, D. A. On the rocks: the microbiology of Antarctic Dry Valley soils. Nature Reviews Microbiology 8, 129-138, doi:10.1038/nrmicro2281 (2010).

28 Zhang, E. et al. Lifting the veil on arid-to-hyperarid Antarctic soil microbiomes: a tale of two oases. Microbiome 8, 37, doi:10.1186/s40168-020-00809-w (2020).

$29 \mathrm{Ji}, \mathrm{M}$. et al. Candidatus Eremiobacterota, a metabolically and phylogenetically diverse terrestrial phylum with acid-tolerant adaptations. Isme J 15, 2692-2707, doi:10.1038/s41396-021-00944-8 (2021). 
30 Montgomery, K. et al. Persistence and resistance: survival mechanisms of Candidatus Dormibacterota from nutrient-poor Antarctic soils. Environmental Microbiology 23, 4276-4294, doi:https://doi.org/10.1111/1462-2920.15610 (2021).

31 Fang, Y. et al. Casting Light on the Adaptation Mechanisms and Evolutionary History of the Widespread Sumerlaeota. mBio 12, e00350-00321, doi:10.1128/mBio.00350-21.

32 Sánchez-Osuna, M., Barbé, J. \& Erill, I. Comparative genomics of the DNA damage-inducible network in the Patescibacteria. Environmental Microbiology 19, 3465-3474, doi:https://doi.org/10.1111/1462-2920.13826 (2017).

33 Bay, S. K. et al. Trace gas oxidizers are widespread and active members of soil microbial communities. Nature Microbiology 6, 246-256, doi:10.1038/s41564-020-00811-w (2021).

34 Li, Q.-M., Zhou, Y.-L., Wei, Z.-F. \& Wang, Y. Phylogenomic insights into distribution and adaptation of Bdellovibrionota in marine waters. bioRxiv, 2020.2011.2001.364414, doi:10.1101/2020.11.01.364414 (2020).

35 Williams, H. N. \& Chen, H. Environmental Regulation of the Distribution and Ecology of Bdellovibrio and Like Organisms. Frontiers in microbiology 11, 545070-545070, doi:10.3389/fmicb.2020.545070 (2020).

36 Imhoff, J. F., Rahn, T., Künzel, S. \& Neulinger, S. C. Photosynthesis Is Widely Distributed among Proteobacteria as Demonstrated by the Phylogeny of PufLM Reaction Center Proteins. Frontiers in microbiology 8, 2679-2679, doi:10.3389/fmicb.2017.02679 (2018).

37 Baker, P. L. et al. A Molecular Biology Tool Kit for the Phototrophic Firmicute Heliobacterium modesticaldum. Applied and environmental microbiology 85, doi:10.1128/aem.01287-19 (2019).

38 Tank, M. \& Bryant, D. A. Nutrient requirements and growth physiology of the photoheterotrophic Acidobacterium, Chloracidobacterium thermophilum. 6, doi:10.3389/fmicb.2015.00226 (2015).

39 Zeng, Y. \& Koblížek, M. in Modern Topics in the Phototrophic Prokaryotes: Environmental and Applied Aspects (ed Patrick C. Hallenbeck) 163-192 (Springer International Publishing, 2017).

40 Zeng, Y. et al. Gemmatimonas groenlandica sp. nov. Is an Aerobic Anoxygenic Phototroph in the Phylum Gemmatimonadetes. 11, doi:10.3389/fmicb.2020.606612 (2021).

41 Zeng, Y., Feng, F., Medová, H., Dean, J. \& Koblížek, M. Functional type 2 photosynthetic reaction centers found in the rare bacterial phylum Gemmatimonadetes. Proceedings of the National Academy of Sciences 111, 7795, doi:10.1073/pnas.1400295111 (2014).

42 Ward, L. M., Li-Hau, F., Kakegawa, T. \& McGlynn, S. E. Complex history of aerobic respiration and phototrophy in the Chloroflexota class Anaerolineae revealed by high-quality draft genome of 
\&lt;em\&gt;Ca\&lt;/em\&gt;. Roseilinea mizusawaensis AA3_104. bioRxiv, 2020.2011.2030.404129, doi:10.1101/2020.11.30.404129 (2020).

43 Thiel, V., Fukushima, S.-I., Kanno, N. \& Hanada, S. in Encyclopedia of Microbiology (Fourth Edition) (ed Thomas M. Schmidt) 651-662 (Academic Press, 2019).

44 Tourna, M., Maclean, P., Condron, L., O'Callaghan, M. \& Wakelin, S. A. Links between sulphur oxidation and sulphur-oxidising bacteria abundance and diversity in soil microcosms based on soxB functional gene analysis. FEMS microbiology ecology 88, 538-549, doi:10.1111/1574-6941.12323 (2014).

45 Anantharaman, K. et al. Expanded diversity of microbial groups that shape the dissimilatory sulfur cycle. The ISME Journa/ 12, 1715-1728, doi:10.1038/s41396-018-0078-0 (2018).

46 Magalhães, C. M., Machado, A., Frank-Fahle, B., Lee, C. K. \& Cary, S. C. The ecological dichotomy of ammonia-oxidizing archaea and bacteria in the hyper-arid soils of the Antarctic Dry Valleys. $\mathbf{5}$, doi:10.3389/fmicb.2014.00515 (2014).

47 Richter, I. et al. Influence of soil properties on archaeal diversity and distribution in the McMurdo Dry Valleys, Antarctica. FEMS Microbiology Ecology 89, 347-359, doi:10.1111/1574-6941.12322 (2014).

48 Ayton, J., Aislabie, J., Barker, G. M., Saul, D. \& Turner, S. Crenarchaeota affiliated with group 1.1b are prevalent in coastal mineral soils of the Ross Sea region of Antarctica. Environmental Microbiology 12, 689-703, doi:https://doi.org/10.1111/j.1462-2920.2009.02111.x (2010).

49 Tolar, B. B. et al. Contribution of ammonia oxidation to chemoautotrophy in Antarctic coastal waters. The ISME Journal 10, 2605-2619, doi:10.1038/ismej.2016.61 (2016).

50 Convey, P. et al. The spatial structure of Antarctic biodiversity. Ecological Monographs 84, 203-244, doi:https://doi.org/10.1890/12-2216.1 (2014).

51 Robinson, S. et al. Rapid change in East Antarctic terrestrial vegetation in response to regional drying. Nature Climate Change 8, 879-884, doi:10.1038/s41558-018-0280-0 (2018).

52 Meier Dimitri, V., Imminger, S., Gillor, O., Woebken, D. \& Lax, S. Distribution of Mixotrophy and Desiccation Survival Mechanisms across Microbial Genomes in an Arid Biological Soil Crust Community. mSystems 6, e00786-00720, doi:10.1128/mSystems.00786-20.

53 Jordaan, K. et al. Hydrogen-Oxidizing Bacteria Are Abundant in Desert Soils and Strongly Stimulated by Hydration. mSystems 5, doi:10.1128/mSystems.01131-20 (2020).

54 Gupta, R. S. \& Khadka, B. J. P. r. Evidence for the presence of key chlorophyll-biosynthesis-related proteins in the genus Rubrobacter (Phylum Actinobacteria) and its implications for the evolution and origin of photosynthesis. 127, 201-218 (2016). 
55 Cardona, T. Origin of Bacteriochlorophyll a and the Early Diversification of Photosynthesis. PLOS ONE 11, e0151250, doi:10.1371/journal.pone.0151250 (2016).

56 Mohammadi, S. S. et al. The Acidophilic Methanotroph Methylacidimicrobium tartarophylax 4AC Grows as Autotroph on H2 Under Microoxic Conditions. 10, doi:10.3389/fmicb.2019.02352 (2019).

57 Søndergaard, D., Pedersen, C. N. S. \& Greening, C. HydDB: A web tool for hydrogenase classification and analysis. Scientific Reports 6, 34212, doi:10.1038/srep34212 (2016).

58 Fritsche, E., Paschos, A., Beisel, H.-G., Böck, A. \& Huber, R. Crystal structure of the hydrogenase maturating endopeptidase HYBD from Escherichia coli11Edited by D. C. Rees. Journal of molecular biology 288, 989-998, doi:https://doi.org/10.1006/jmbi.1999.2719 (1999).

59 Schäfer, C. et al. Structure of an Actinobacterial-Type [NiFe]-Hydrogenase Reveals Insight into 02Tolerant H2 Oxidation. Structure 24, 285-292, doi:https://doi.org/10.1016/j.str.2015.11.010 (2016).

60 Waterhouse, A. et al. SWISS-MODEL: homology modelling of protein structures and complexes. Nucleic Acids Research 46, W296-W303, doi:10.1093/nar/gky427 (2018).

61 Dobbek, H., Gremer, L., Kiefersauer, R., Huber, R. \& Meyer, O. Catalysis at a dinuclear [CuSMo(\&lt;img src=\&quot;pending:yes\&quot; l:ref-type=\&quot;journal\&quot; hwp:journal=\&quot;pnas\&quot; hwp:volume=\&quot;99\&quot; hwp:issue=\&quot;25\&quot; hwp:article=\&quot; 15971\&quot; l:sub-ref=\&quot;inline-graphic-1\&quot; l:type=\&quot;image/*\&quot; class=\&quot;inline-graphic\&quot; alt=\&quot;Graphic\&quot;/\&gt;0)OH] cluster in a CO dehydrogenase resolved at 1.1-Å resolution. Proceedings of the National Academy of Sciences 99, 15971, doi:10.1073/pnas.212640899 (2002).

62 Montgomery, K. et al. Persistence and resistance: survival mechanisms of Candidatus Dormibacterota from nutrient-poor Antarctic soils. Environmental Microbiology n/a, doi:https://doi.org/10.1111/1462-2920.15610 (2021).

$63 \mathrm{Ji}, \mathrm{M}$. et al. Candidatus Eremiobacterota, a metabolically and phylogenetically diverse terrestrial phylum with acid-tolerant adaptations. The ISME Journal, doi:10.1038/s41396-021-00944-8 (2021).

64 Guo, R. \& Conrad, R. Extraction and characterization of soil hydrogenases oxidizing atmospheric hydrogen. Soil Biology and Biochemistry 40, 1149-1154, doi:https://doi.org/10.1016/j.soilbio.2007.12.007 (2008).

65 Khdhiri, M. et al. Soil carbon content and relative abundance of high affinity H2-oxidizing bacteria predict atmospheric $\mathrm{H} 2$ soil uptake activity better than soil microbial community composition. Soil Biology and Biochemistry 85, 1-9, doi:https://doi.org/10.1016/j.soilbio.2015.02.030 (2015).

66 Piché-Choquette, S., Tremblay, J., Tringe, S. G. \& Constant, P. H2-saturation of high affinity H2oxidizing bacteria alters the ecological niche of soil microorganisms unevenly among taxonomic groups. 
PeerJ 4, e1782-e1782, doi:10.7717/peerj.1782 (2016).

67 Ortiz, M. et al. A genome compendium reveals diverse metabolic adaptations of Antarctic soil microorganisms. bioRxiv, 2020.2008.2006.239558, doi:10.1101/2020.08.06.239558 (2020).

68 Constant, P., Chowdhury, S. P., Pratscher, J. \& Conrad, R. Streptomycetes contributing to atmospheric molecular hydrogen soil uptake are widespread and encode a putative high-affinity [NiFe]hydrogenase. Environ Microbio/ 12, 821-829, doi:10.1111/j.1462-2920.2009.02130.x (2010).

69 Finstad, K. M. et al. Microbial Community Structure and the Persistence of Cyanobacterial Populations in Salt Crusts of the Hyperarid Atacama Desert from Genome-Resolved Metagenomics. 8, doi:10.3389/fmicb.2017.01435 (2017).

70 Jung, P. et al. Water availability shapes edaphic and lithic cyanobacterial communities in the Atacama Desert. Journal of Phycology 55, 1306-1318, doi:https://doi.org/10.1111/jpy.12908 (2019).

71 Lee, K. C. et al. Stochastic and Deterministic Effects of a Moisture Gradient on Soil Microbial Communities in the McMurdo Dry Valleys of Antarctica. Front Microbio/ 9, 2619-2619, doi:10.3389/fmicb.2018.02619 (2018).

72 Leung, P. M. et al. Energetic Basis of Microbial Growth and Persistence in Desert Ecosystems. mSystems 5, e00495-00419, doi:10.1128/mSystems.00495-19 (2020).

73 Tebo, B. M. et al. Microbial communities in dark oligotrophic volcanic ice cave ecosystems of Mt. Erebus, Antarctica. Front Microbio/ 6, 179-179, doi:10.3389/fmicb.2015.00179 (2015).

74 Tamura, T. et al. Description of Actinomycetospora chibensis sp. nov., Actinomycetospora chlora sp. nov., Actinomycetospora cinnamomea sp. nov., Actinomycetospora corticicola sp. nov., Actinomycetospora lutea sp. nov., Actinomycetospora straminea sp. nov. and Actinomycetospora succinea sp. nov. and emended description. International Journal of Systematic and Evolutionary Microbiology 61, 1275-1280, doi:10.1099/ijs.0.024166-0 (2011).

75 Yamamura, H., Tamura, T., Sakiyama, Y. \& Harayama, S. Nocardia amamiensis sp. nov., isolated from a sugar-cane field in Japan. International journal of systematic and evolutionary microbiology $\mathbf{5 7}$, 1599, doi:10.1099/ijs.0.64829-0 (2007).

76 Fang, B. Z. et al. Nocardia aurea sp. nov., a novel actinobacterium isolated from a karstic subterranean environment. International Journal of Systematic and Evolutionary Microbiology 69, 159164, doi:10.1099/ijsem.0.003122 (2019).

77 Jurado, V. et al. Nocardia altamirensis sp. nov., isolated from Altamira cave, Cantabria, Spain. International journal of systematic and evolutionary microbiology 58, 2210, doi:10.1099/ijs.0.65482-0 (2008). 
78 Demaree, J. \& Smith, N. Nocardia vaccinii n. sp. causing galls on Blue-berry plants. Phytopathology 42 (1952).

$79 \mathrm{Li}, \mathrm{X}$. et al. Actinocorallia populi sp. nov., an endophytic actinomycete isolated from a root of Populus adenopoda (Maxim.). International journal of systematic and evolutionary microbiology 68, 2325, doi:10.1099/ijsem.0.002840 (2018).

80 Golovacheva, R. \& Karavaĭko, G. J. M. Sulfobacillus, a new genus of thermophilic sporulating bacteria. $47,815-822(1978)$.

81 Schorn, M. A. et al. Sequencing rare marine actinomycete genomes reveals high density of unique natural product biosynthetic gene clusters. Microbiology (Reading, England) 162, 2075, doi:10.1099/mic.0.000386 (2016).

82 Klenk, H.-P. et al. Genome sequence of the ocean sediment bacterium Saccharomonospora marina type strain (XMU15(T)). Standards in genomic sciences 6, 265, doi:10.4056/sigs.2655905 (2012).

83 Liu, Z. P., Wu, J. F., Liu, Z. H. \& Liu, S. J. Pseudonocardia ammonioxydans sp. nov., isolated from coastal sediment. Int J Syst Evol Microbio/ 56, 555-558, doi:10.1099/ijs.0.63878-0 (2006).

84 Tian, X.-P. et al. Streptomyces nanshensis sp. nov., isolated from the Nansha Islands in the South China Sea. International journal of systematic and evolutionary microbiology 59, 745, doi:10.1099/ijs.0.003442-0 (2009).

85 Maker, A., Hemp, J., Pace, L. A., Ward, L. M. \& Fischer, W. W. Draft Genome Sequence of Hydrogenibacillus schlegelii MA48, a Deep-Branching Member of the Bacilli Class of Firmicutes. Genome announcements 5, e00380-00316, doi:10.1128/genomeA.00380-16 (2017).

86 Niu, M.-M. et al. Amycolatopsis nivea sp. nov., isolated from a Yellow River sample. 70, 30843090, doi:https://doi.org/10.1099/ijsem.0.004134 (2020).

87 Torkko, P. et al. Mycobacterium palustre sp. nov., a potentially pathogenic, slowly growing mycobacterium isolated from clinical and veterinary specimens and from Finnish stream waters. International journal of systematic and evolutionary microbiology 52, 1519, doi:10.1099/00207713-52-51519 (2002).

88 Albuquerque, L. et al. Meiothermus rufus sp. nov., a new slightly thermophilic red-pigmented species and emended description of the genus Meiothermus. Syst App/ Microbio/ 32, 306-313, doi:10.1016/j.syapm.2009.05.002 (2009).

89 Chung, A. P., Rainey, F., Nobre, M. F., Burghardt, J. \& Costa, M. S. D. Meiothermus cerbereus sp. nov., a New Slightly Thermophilic Species with High Levels of 3-Hydroxy Fatty Acids. 47, 1225-1230, doi:https://doi.org/10.1099/00207713-47-4-1225 (1997). 
90 Yoshida, M. et al. Draft Genome Sequence of sp. Strain shizuoka-1, a Novel Mycobacterium Isolated from Groundwater of a Bathing Facility in Shizuoka, Japan. Genome announcements 5, doi:10.1128/genomeA.01309-17 (2017).

91 Trujillo, M. E. \& Goodfellow, M. Polyphasic taxonomic study of clinically significant actinomadurae including the description of Actinomadura latina sp. nov. Zentralblatt fur Bakteriologie 285, 212-233, doi:10.1016/S0934-8840(97)80029-1 (1997).

92 Phelippeau, M., Croce, O., Robert, C., Raoult, D. \& Drancourt, M. Draft genome sequence of Mycobacterium lentiflavum CSUR P1491. Genome Announcements 3, doi:10.1128/genomeA.00817-15 (2015).

93 Richter, E., Niemann, S., Gloeckner, F. O., Pfyffer, G. E. \& Rüsch-Gerdes, S. Mycobacterium holsaticum sp. nov. Int J Syst Evol Microbio/ 52, 1991-1996, doi:10.1099/00207713-52-6-1991 (2002).

94 Turenne, C. Y. et al. Mycobacterium saskatchewanense sp. nov., a novel slowly growing scotochromogenic species from human clinical isolates related to Mycobacterium interjectum and Accuprobe-positive for Mycobacterium avium complex. Int J Syst Evol Microbio/ 54, 659-667, doi:10.1099/ijs.0.02739-0 (2004).

95 Ustinova, V. et al. First Draft Genome Sequence of a Mycobacterium gordonae Clinical Isolate. Genome Announcements 4, doi:10.1128/genomeA.00638-16 (2016).

96 Tortoli, E. et al. Mycobacterium tusciae sp. nov. International journal of systematic bacteriology 49 Pt 4, 1839-1844, doi:10.1099/00207713-49-4-1839 (1999).

97 Ezeoke, l. et al. Nocardia amikacinitolerans sp. nov., an amikacinresistant human pathogen. International Journal of Systematic and Evolutionary Microbiology 63, 1056-1061, doi:10.1099/ijs.0.039990-0 (2013).

98 Nakazawa, A. et al. [A case of pulmonary Mycobacterium gordonae infection diagnosed by gastric juice culture and successfully treated with multidrug chemotherapy]. Kekkaku : [Tuberculosis] 87, 727-731 (2012).

99 Hashemi Shahraki, A. et al. Mycobacterium aquaticum sp. nov., a rapidly growing species isolated from haemodialysis water. International Journal of Systematic and Evolutionary Microbiology 67, 32793282, doi:https://doi.org/10.1099/ijsem.0.002103 (2017).

100 Bolger, A. M., Lohse, M. \& Usadel, B. Trimmomatic: a flexible trimmer for Illumina sequence data. Bioinformatics (Oxford, England) 30, 2114, doi:10.1093/bioinformatics/btu170 (2014).

101 Li, D., Liu, C. M., Luo, R., Sadakane, K. \& Lam, T. MEGAHIT: an ultra-fast single-node solution for large and complex metagenomics assembly via succinct de Bruijn graph. Bioinformatics 31, 1674-1676, doi:10.1093/bioinformatics/btv033 (2015). 
103 Wu, Y.-W., Tang, Y.-H., Tringe, S. G., Simmons, B. A. \& Singer, S. W. MaxBin: an automated binning method to recover individual genomes from metagenomes using an expectation-maximization algorithm. Microbiome 2, 26, doi:10.1186/2049-2618-2-26 (2014).

104 Kang, D. D., Froula, J., Egan, R. \& Wang, Z. MetaBAT, an efficient tool for accurately reconstructing single genomes from complex microbial communities. PeerJ 3, e1165-e1165, doi:10.7717/peerj.1165 (2015).

105 Kang, D. D. et al. MetaBAT 2: an adaptive binning algorithm for robust and efficient genome reconstruction from metagenome assemblies. PeerJ 7, e7359-e7359, doi:10.7717/peerj.7359 (2019).

106 Parks, D. H., Imelfort, M., Skennerton, C. T., Hugenholtz, P. \& Tyson, G. W. CheckM: assessing the quality of microbial genomes recovered from isolates, single cells, and metagenomes. Genome Res 25, 1043-1055, doi:10.1101/gr.186072.114 (2015).

107 Chaumeil, P.-A., Mussig, A. J., Hugenholtz, P. \& Parks, D. H. GTDB-Tk: a toolkit to classify genomes with the Genome Taxonomy Database. Bioinformatics 36, 1925-1927, doi:10.1093/bioinformatics/btz848 (2020).

108 Olm, M. R., Brown, C. T., Brooks, B. \& Banfield, J. F. dRep: a tool for fast and accurate genomic comparisons that enables improved genome recovery from metagenomes through de-replication. The ISME journal 11, 2864-2868, doi:10.1038/ismej.2017.126 (2017).

109 Finn, R. D. et al. Pfam: the protein families database. Nucleic acids research 42, D222-D230, doi:10.1093/nar/gkt1223 (2014).

110 Camacho, C. et al. BLAST+: architecture and applications. BMC Bioinformatics 10, 421, doi:10.1186/1471-2105-10-421 (2009).

111 The UniProt, C. UniProt: the universal protein knowledgebase in 2021. Nucleic Acids Research 49, D480-D489, doi:10.1093/nar/gkaa1100 (2021).

112 Buchfink, B., Reuter, K. \& Drost, H.-G. Sensitive protein alignments at tree-of-life scale using DIAMOND. Nature Methods 18, 366-368, doi:10.1038/s41592-021-01101-x (2021).

113 Darling, A. E. et al. PhyloSift: phylogenetic analysis of genomes and metagenomes. PeerJ 2, e243, doi:10.7717/peerj.243 (2014).

114 Wickham, H. ggplot2: Elegant Graphics for Data Analysis. (Springer New York, 2009).

$115 \mathrm{Li}, \mathrm{H}$. Minimap2: pairwise alignment for nucleotide sequences. Bioinformatics 34, 3094-3100, doi:10.1093/bioinformatics/bty191 (2018). 
116 Menzel, P., Ng, K. L. \& Krogh, A. Fast and sensitive taxonomic classification for metagenomics with Kaiju. Nature Communications 7, 11257, doi:10.1038/ncomms11257 (2016).

117 Lombard, V., Golaconda Ramulu, H., Drula, E., Coutinho, P. M. \& Henrissat, B. The carbohydrateactive enzymes database (CAZy) in 2013. Nucleic Acids Res 42, D490-495, doi:10.1093/nar/gkt1178 (2014).

118 Jones, P. et al. InterProScan 5: genome-scale protein function classification. Bioinformatics 30, 1236-1240, doi:10.1093/bioinformatics/btu031 (2014).

119 Krogh, A., Larsson, B., von Heijne, G. \& Sonnhammer, E. L. Predicting transmembrane protein topology with a hidden Markov model: application to complete genomes. Journal of molecular biology 305, 567-580, doi:10.1006/jmbi.2000.4315 (2001).

120 Katoh, K., Misawa, K., Kuma, K. i. \& Miyata, T. J. N. a. r. MAFFT: a novel method for rapid multiple sequence alignment based on fast Fourier transform. 30, 3059-3066 (2002).

121 Katoh, K. \& Standley, D. M. MAFFT multiple sequence alignment software version 7: improvements in performance and usability. Molecular biology and evolution 30,772-780, doi:10.1093/molbev/mst010 (2013).

122 Capella-Gutiérrez, S., Silla-Martínez, J. \& Gabaldón, T. trimAl: a tool for automated alignment trimming in large-scale phylogenetic analyses. Bioinformatics 25, 1972-1973, doi:10.1093/bioinformatics/btp348 (2009).

123 Nguyen, L.-T., Schmidt, H. A., von Haeseler, A. \& Minh, B. Q. IQ-TREE: a fast and effective stochastic algorithm for estimating maximum-likelihood phylogenies. Molecular biology and evolution 32, 268-274, doi:10.1093/molbev/msu300 (2015).

124 Guindon, S. et al. New Algorithms and Methods to Estimate Maximum-Likelihood Phylogenies: Assessing the Performance of PhyML 3.0. Systematic Biology 59, 307-321, doi:10.1093/sysbio/syq010 (2010).

125 Letunic, I. \& Bork, P. Interactive tree of life (iTOL) v3: an online tool for the display and annotation of phylogenetic and other trees. Nucleic Acids Res 44, W242-245, doi:10.1093/nar/gkw290 (2016).

126 Gasteiger, E. et al. ExPASy: The proteomics server for in-depth protein knowledge and analysis. Nucleic Acids Res 31, 3784-3788, doi:10.1093/nar/gkg563 (2003).

127 Blum, M. et al. The InterPro protein families and domains database: 20 years on. Nucleic Acids Res 49, D344-d354, doi:10.1093/nar/gkaa977 (2021).

\section{Figures}



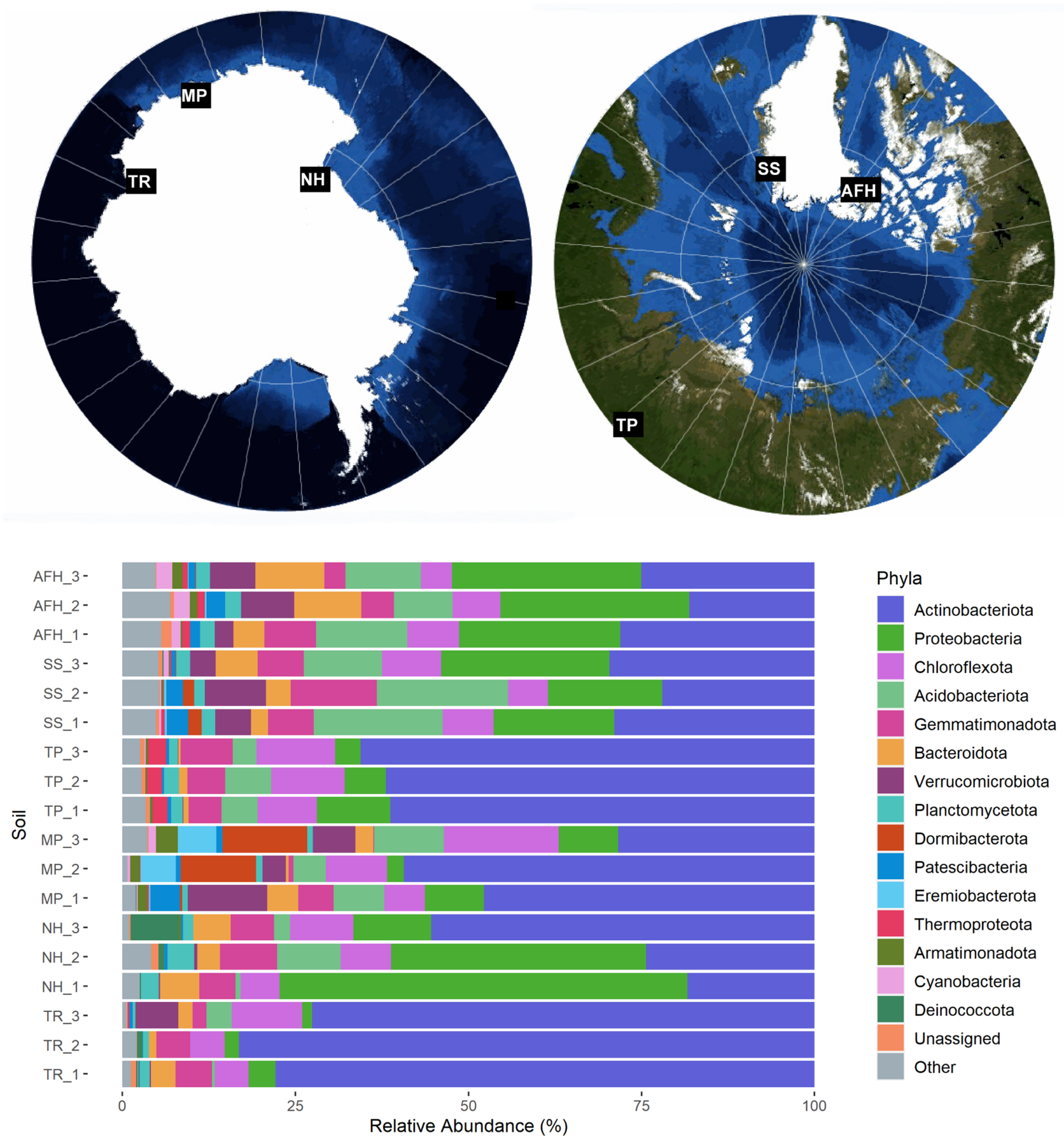

\section{Figure 1}

Community composition of the 18 global desert soils, classified using the universal single-copy ribosomal protein gene rpIP retrieved from shotgun metagenomic reads. The relative abundance of major bacterial and archaeal phyla residing in triplicate desert soils from Alexandra Fjord Highlands (AFH), Spitsbergen Svalbard (SS), Tibetan Plateau (TP), Mitchell Peninsula (MP), New Harbour (NH) and The Ridge (TR) are displayed; phyla with less than $2 \%$ relative abundance in all soil samples were grouped to 
the "Other" phyla. Actinobacteriota dominate all sites, particularly TR (average 77.9\%), TP (average 62.9 $\%$ ) and MP (average $45.2 \%$ ). Photosynthetic Cyanobacteria are extremely scarce within NH, TP and TR samples $(<0.07 \%)$, with greater average abundances observed at MP $(0.6 \%)$, SS $(0.4 \%)$ and AFH $(2.0 \%)$. $\mathrm{Ca}$. Eremiobacterota and Ca. Dormibacterota dominate MP microbiomes (average $7.8 \%$ and $3.6 \%$, respectively) and are present at lower levels within SS and AFH. Archaea are minor members of these ecosystems (average relative abundances; $<0.2 \%$ within TR, MP, NH; $2.6 \%$ within TP; $0.5 \%$ within SS; $1.2 \%$ within $\mathrm{AFH})$.

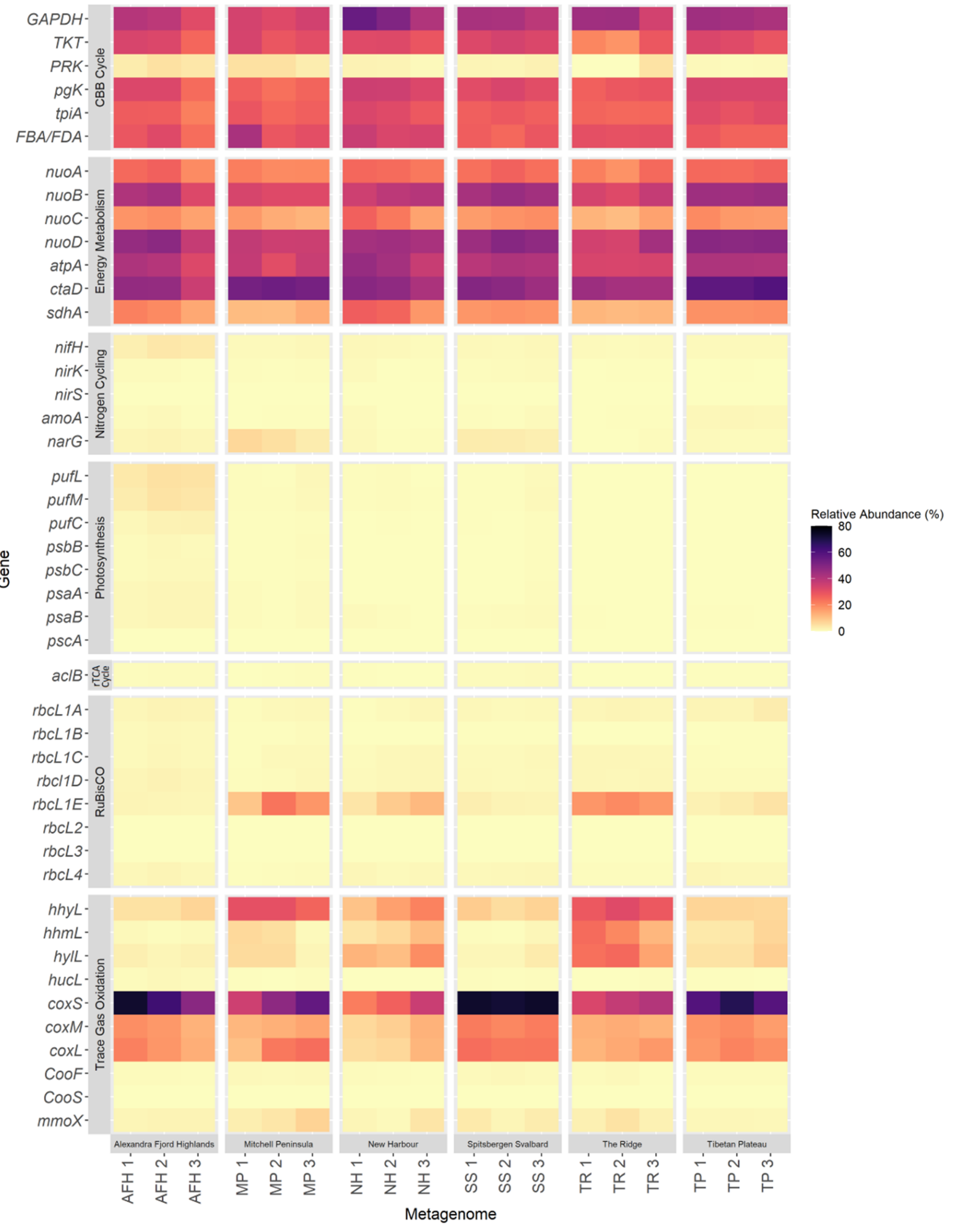


Figure 2

Heatmap displaying the abundance of key metabolic marker genes involved in carbon fixation and energy conservation, and their distribution throughout 18 metagenomes spanning six cold desert regions. For each region, relative gene abundances were displayed for three metagenomes. Genes encoding the CBB cycle, energy metabolism, aerobic respiration, nitrogen cycling, and trace gas oxidation were widely distributed throughout all environments. Phototrophy genes were more abundant within Alexandra Fjord Highland metagenomes than the other sites studied.

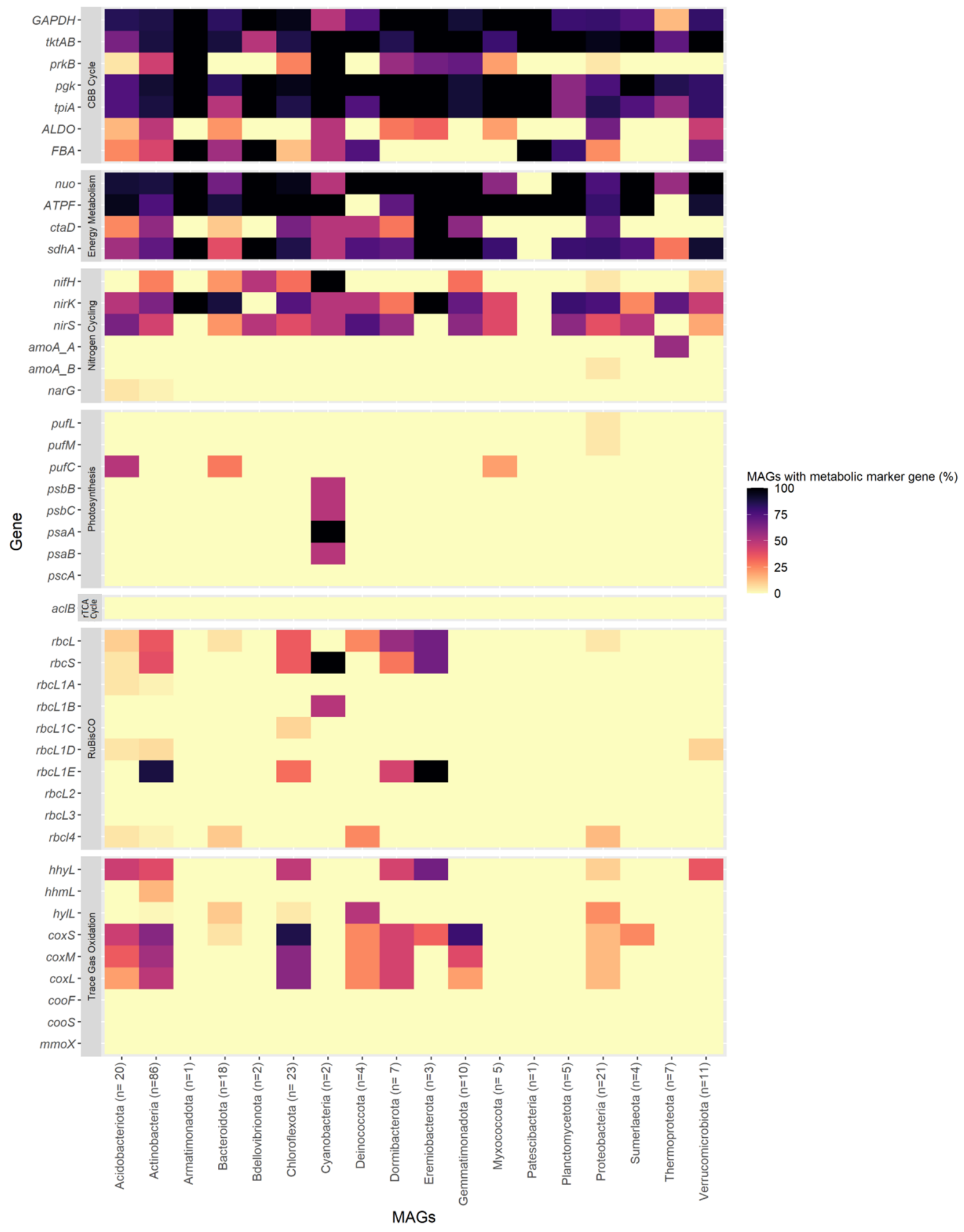




\section{Figure 3}

Heatmap displaying key functional genes involved in microbial autotrophy and energy conservation, and their distribution throughout the 76 high-quality ( $>90 \%$ completeness, $<5 \%$ contamination) and 154 medium-quality bins (50-90\% completeness, 5-10\% contamination) constructed. Abundances are displayed relative to the total number of MAGs from each phylogenetic group. Genes encoding the CBB cycle, energy metabolism, respiration, and nitrogen cycling were widely distributed. Phototrophy genes were primarily limited to Cyanobacteria MAGs, whilst trace gas oxidation genes were widely distributed throughout 11 of the 18 phyla detected through MAG construction.

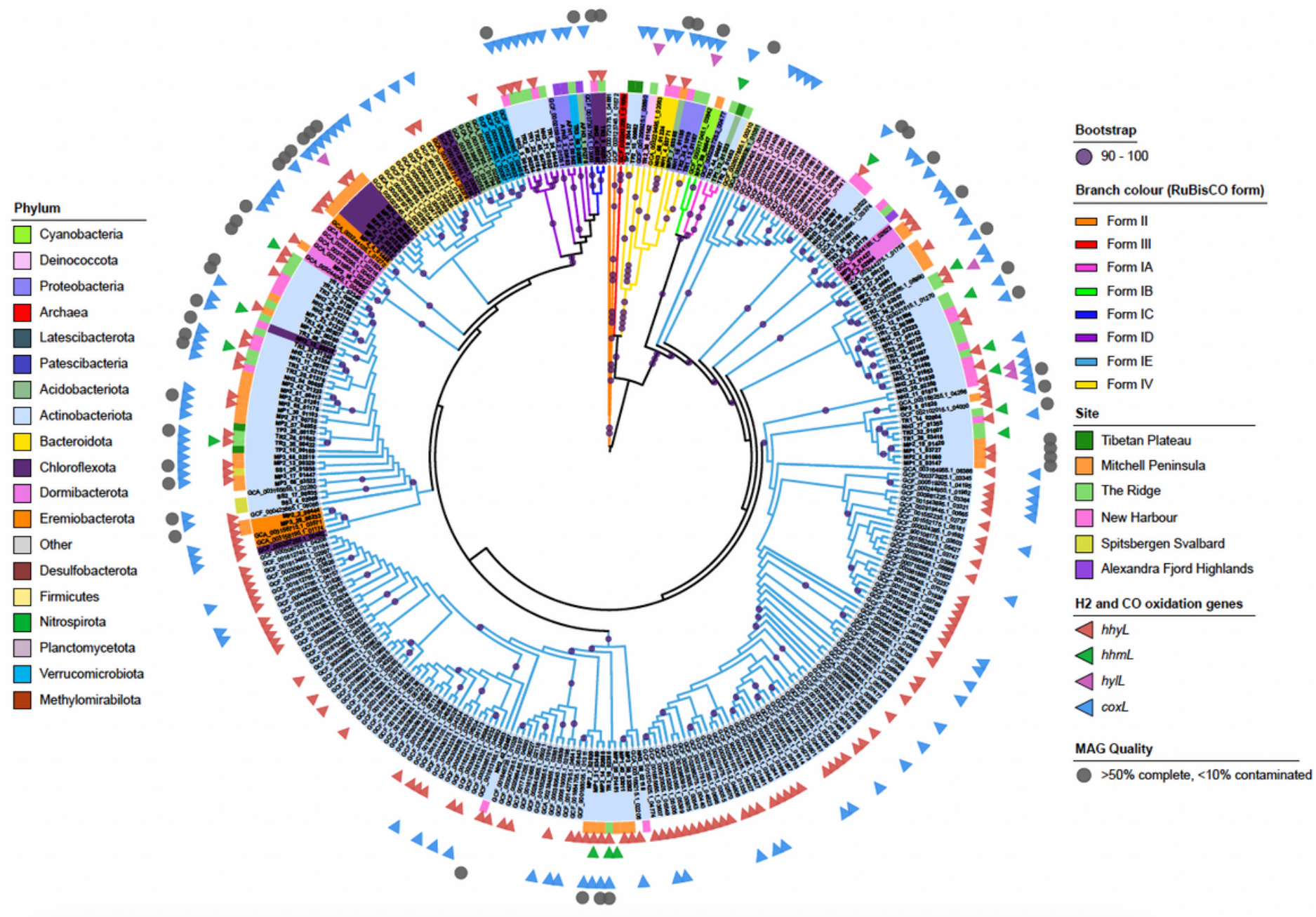

\section{Figure 4}

Maximum likelihood phylogenetic tree of RuBisCO gene sequences focusing on form IE, pruned from a larger tree containing binned cold desert metagenomic assembled genomes (MAGs) and over 3000 published genomes. Leaves are coloured to represent phylum, while coloured branches show RuBisCO form. The cold desert-site that each MAG was obtained from is shown in the outer ring. Genomes which additionally harboured high affinity groups $1 \mathrm{~h}$-[NiFe]-hydrogenase (), 1m-[NiFe]-hydrogenase (), 11-[NiFe]hydrogenase () and/or aerobic carbon monoxide dehydrogenase () with an active-site loop are indicated by outer triangles, coloured red, green, pink, and blue, respectively. Bootstrap values \&gt; $90 \%$ are depicted 
as filled circles on branches. High-quality MAGS constructed in this study are marked with grey circles. RuBisCO form IE is highly diverse, spanning 8 bacterial phyla (Actinobacteriota, Chloroflexota, Firmicutes, Verrucomicrobiota, C. Dormibacterota, C. Eremiobacterota, Acidobacteriota and Deinococcota) with multiple distinct clades observed. Most genomes containing RuBisCO form IE also contained high-affinity group 1-[NiFe]-hydrogenase and/or aerobic carbon monoxide dehydrogenase.

A
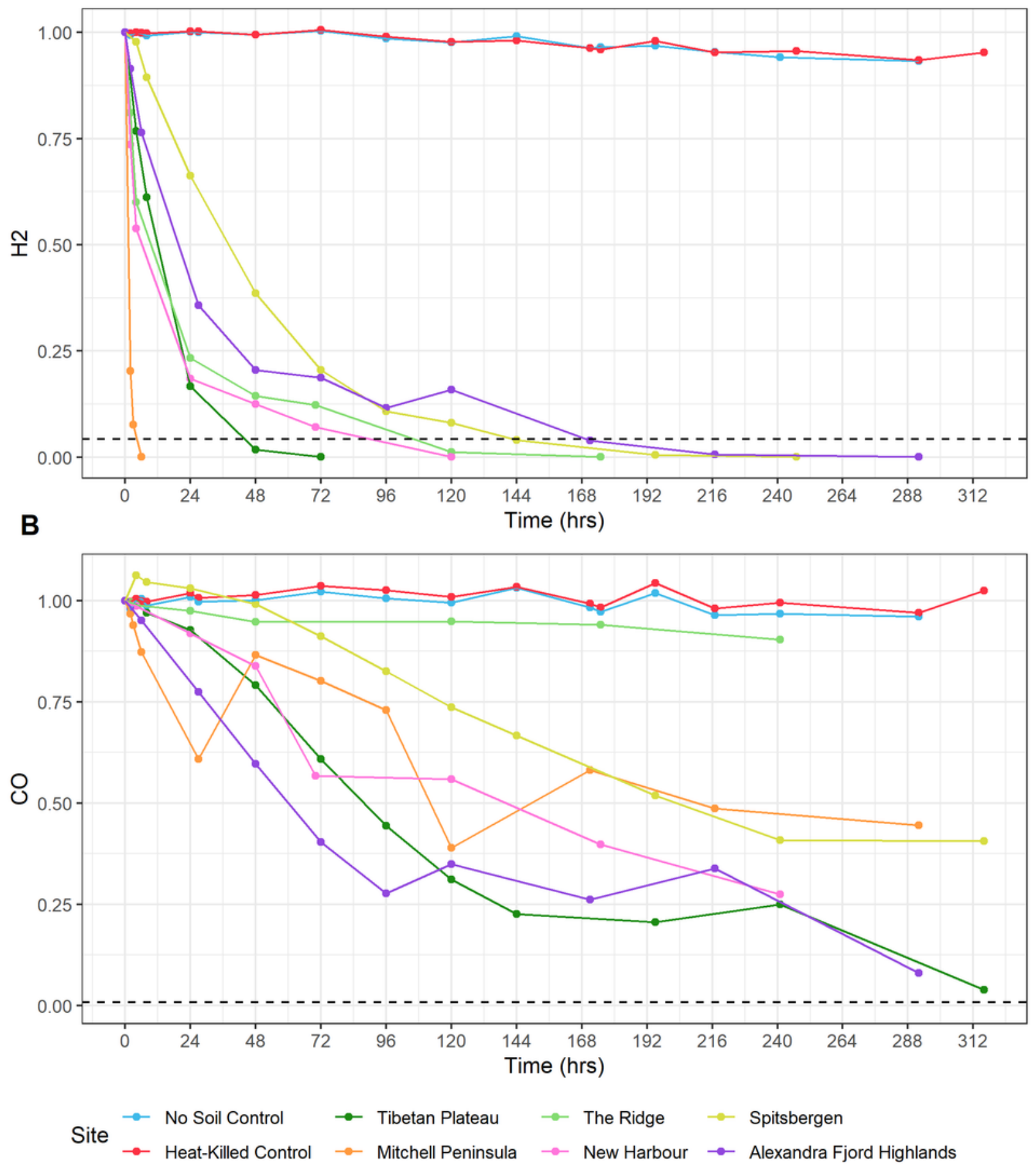

Figure 5 
The consumption and oxidation of atmospheric A hydrogen and B carbon monoxide by surface soil microcosms from six cold deserts. Values shown have been normalised against the starting concentration of each gas and are the mean of biological triplicates. The dashed line in A indicates atmospheric $\mathrm{H} 2$ (530 p.p.b.v) and in B indicates atmospheric $\mathrm{CO}$ (90 p.p.b.v). Rapid high-affinity hydrogenase activity was observed across all sites (Average $\mathrm{H} 2$ consumption; MP $421.4 \mathrm{nmol} / \mathrm{mol} / \mathrm{hr} / \mathrm{g}$, $\mathrm{NH} 42.6 \mathrm{nmol} / \mathrm{mol} / \mathrm{hr} / \mathrm{g}$, TR $41.1 \mathrm{nmol} / \mathrm{mol} / \mathrm{hr} / \mathrm{g}$, TP $35.6 \mathrm{nmol} / \mathrm{mol} / \mathrm{hr} / \mathrm{g}$, AFH $21.6 \mathrm{nmol} / \mathrm{mol} / \mathrm{hr} / \mathrm{g}$, SS $9.4 \mathrm{nmol} / \mathrm{mol} / \mathrm{hr} / \mathrm{g}$ ), particularly within the Mitchell Peninsula microcosms, each of which consumed hydrogen to sub-atmospheric levels within 6 hours of incubation. Carbon monoxide consumption was observed however, compared to hydrogen consumption, these rates were slower and varied greatly between samples within each site. 

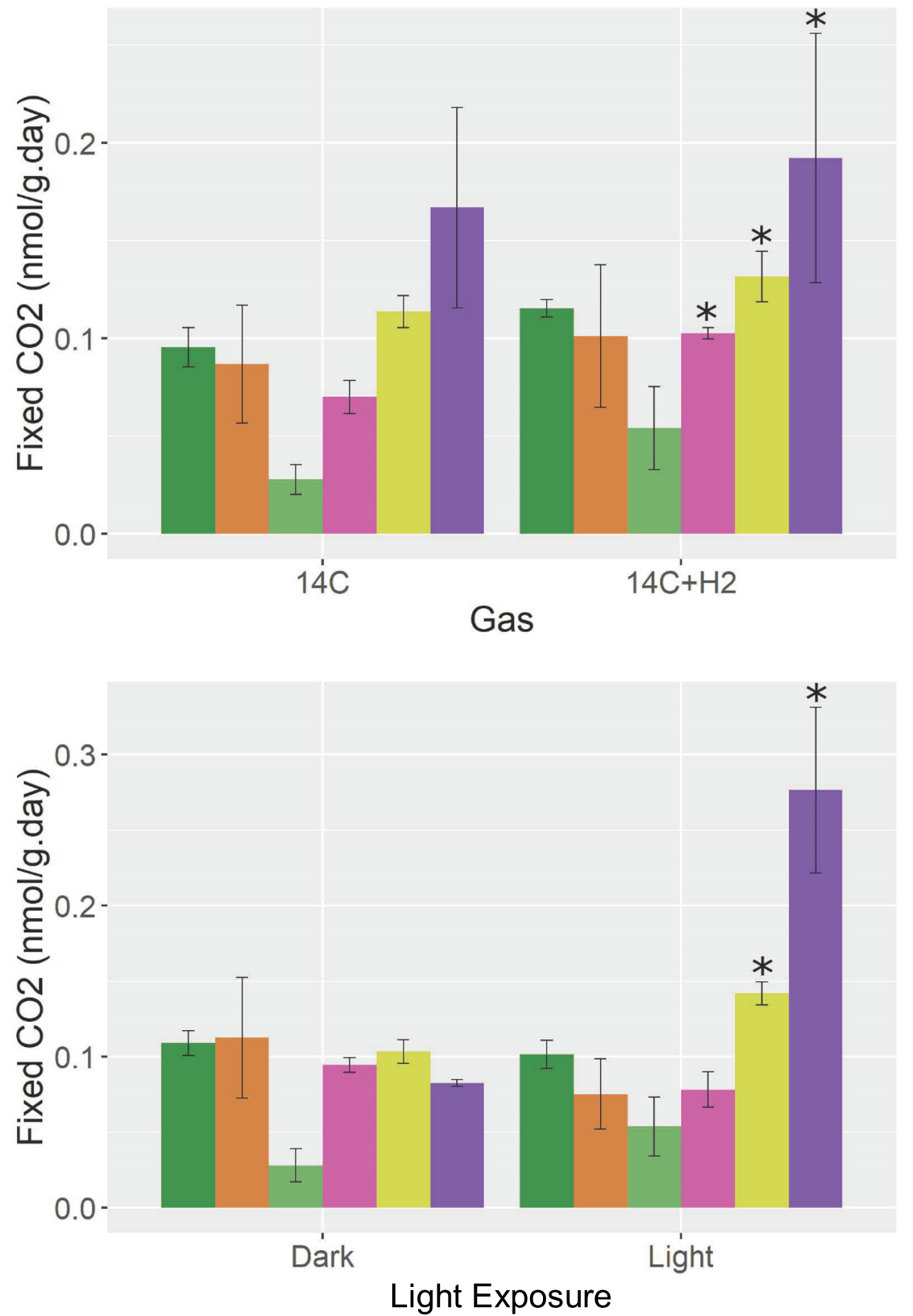

\section{Desert}

Tibetan Plateau Mitchell Peninsula The Ridge New Harbour Spitsbergen Svalbard Alexandra Fjord Highlands

\section{Figure 6}

The increase in 14CO2 assimilation in soils from six cold deserts under A) hydrogen stimulation and B) light exposure. Carbon assimilation by soil microcosms from all deserts were consistently stimulated by the addition of atmospherically relevant hydrogen concentrations ( 10 p.p.m.v), with significant increases observed within microcosms from New Harbour $(p=0.013)$, Spitsbergen Svalbard $(p=0.033)$ and Alexandra Fjord Highlands $(p=0.031)$. Light exposure also led to a significant increase in carbon assimilation within soils from Spitsbergen Svalbard and Alexandra Fjord Highlands $(p=0.022$ and 0.015 , respectively), but did not significantly influence primary production across the other deserts. Normality 
was determined using Shapiro-Wilk tests. When the data was normally distributed, statistical significance was determined using a two-tailed paired $T$ test. Otherwise, a two-tailed Wilcoxon signed-rank test with a Bonferroni correction was implemented.

\section{Supplementary Files}

This is a list of supplementary files associated with this preprint. Click to download.

- Supplementary1.xlsx

- Supplementary2.xlsx

- Supplementary3.xlsx

- Supplementary4.xlsx

- Supplementary5.csv

- Supplementary6.xlsx

- Supplementary7.xlsx

- Supplementary8.xlsx

- Supplementary9.xlsx

- Supplementary10.xlsx

- Supplementary11.xlsx

- Supplementary12.pdf

- Supplementary13.png

- Supplementary14.pdf

- Supplementary15.xIsx

- Supplementary16.png

- Supplementary17.png

- Supplementary18.docx

- Supplementary19.tif 